\title{
The tidal evolution of dark matter substructure - II. The impact of artificial disruption on subhalo mass functions and radial profiles
}

\author{
Sheridan B. Green ${ }^{\oplus},{ }^{1 \star} \nmid$ Frank C. van den Bosch ${ }^{\oplus 1,2}$ and Fangzhou Jiang ${ }^{\oplus 3,4} \ddagger$ \\ ${ }^{1}$ Department of Physics, Yale University, P.O. Box 208120, New Haven, CT 06520-8120, USA \\ ${ }^{2}$ Department of Astronomy, Yale University, P.O. Box 208101, New Haven, CT 06520-8101, USA \\ ${ }^{3}$ TAPIR, California Institute of Technology, Pasadena, CA 91125, USA \\ ${ }^{4}$ Carnegie Observatories, 813 Santa Barbara Street, Pasadena, CA 91101, USA
}

Accepted 2021 February 23. Received 2021 February 12; in original form 2020 December 18

\begin{abstract}
Several recent studies have indicated that artificial subhalo disruption (the spontaneous, non-physical disintegration of a subhalo) remains prevalent in state-of-the-art dark matter (DM)-only cosmological simulations. In order to quantify the impact of disruption on the inferred subhalo demographics, we augment the semi-analytical SatGen dynamical subhalo evolution model with an improved treatment of tidal stripping that is calibrated using the Dynamical Aspects of SubHaloes database of idealized highresolution simulations of subhalo evolution, which are free from artificial disruption. We also develop a model of artificial disruption that reproduces the statistical properties of disruption in the Bolshoi simulation. Using this framework, we predict subhalo mass functions (SHMFs), number density profiles, and substructure mass fractions and study how these quantities are impacted by artificial disruption and mass resolution limits. We find that artificial disruption affects these quantities at the 10-20 per cent level, ameliorating previous concerns that it may suppress the SHMF by as much as a factor of 2 . We demonstrate that semi-analytical substructure modelling must include orbit integration in order to properly account for splashback haloes, which make up roughly half of the subhalo population. We show that the resolution limit of $N$-body simulations, rather than artificial disruption, is the primary cause of the radial bias in subhalo number density found in DM-only simulations. Hence, we conclude that the mass resolution remains the primary limitation of using such simulations to study subhaloes. Our model provides a fast, flexible, and accurate alternative to studying substructure statistics in the absence of both numerical resolution limits and artificial disruption.
\end{abstract}

Key words: methods: numerical-galaxies: haloes - dark matter.

\section{INTRODUCTION}

The standard Lambda cold dark matter $(\Lambda \mathrm{CDM})$ cosmological model predicts that structure forms as the consequence of primordial dark matter (DM) overdensities that collapse to form self-bound haloes. Smaller perturbations collapse earlier and merge to form larger haloes, resulting in a hierarchical halo assembly process that spans all mass scales. By studying halo evolution via cosmological $N$-body simulations, it is clear that the tightly bound central regions of smaller haloes survive the merger process, persisting as orbiting subhaloes within the treacherous environment of their host halo, where they are subjected to dynamical friction (DF) and disruptive tidal forces (e.g. Mo, van den Bosch \& White 2010). Neglecting the impact of baryonic physics, this merger process is roughly self-similar due to the scalefree nature of gravitational collapse, ultimately resulting in an entire hierarchy of substructure, where subhaloes themselves host subsubhaloes, and so on all the way down (Tormen, Bouchet \& White 1997; Gao et al. 2004; Kravtsov et al. 2004; Giocoli et al. 2010).

\footnotetext{
${ }^{\star}$ E-mail: sheridan.green@yale.edu

$\dagger$ NSF Graduate Research Fellow.

$\ddagger$ Troesh Scholar.
}

The population statistics of DM substructure are most often summarized in terms of subhalo mass functions (SHMFs) and radial profiles; these summary statistics depend heavily on the underlying particle nature of DM. For example, the free-streaming cutoff scale, set by the DM thermal velocity, impacts the lowmass end of the SHMF (e.g. Knebe et al. 2008; Lovell et al. 2014; Colín et al. 2015; Bose et al. 2017), while non-negligible DM self-interactions result in cored inner halo density profiles (e.g. Burkert 2000; Vogelsberger, Zavala \& Loeb 2012; Rocha et al. 2013), which impacts the survivability of substructure in the presence of tides (e.g. Peñarrubia et al. 2010). The predictions of substructure demographics made by these various DM models differ primarily at the low mass end. Consequently, many observational searches are underway in the attempt to constrain the abundance of low mass substructure, leveraging gravitational lensing (e.g. Dalal \& Kochanek 2002; Keeton \& Moustakas 2009; Vegetti et al. 2014; Shu et al. 2015; Hezaveh et al. 2016; Gilman, Birrer \& Treu 2020; Vattis, Toomey \& Koushiappas 2020), indirect detection via DM annihilation to $\gamma$ rays or decay signals (e.g. Strigari et al. 2007; Pieri, Bertone \& Branchini 2008; Hayashi et al. 2016; Hiroshima, Ando \& Ishiyama 2018; Delos 2019; Facchinetti, Lavalle \& Stref 2020; Rico 2020; Somalwar et al. 2021), and gaps in stellar streams (e.g. Carlberg 2012; Ngan \& Carlberg 2014; Erkal et al. 2016; Bonaca et al. 2020; 
Necib et al. 2020), among other approaches. Since satellite galaxies are inferred to live within subhaloes, with their respective properties related via the galaxy-halo connection, DM substructure statistics are intimately connected to satellite galaxy abundances (e.g. Vale \& Ostriker 2006; Behroozi, Wechsler \& Conroy 2013; Hearin et al. 2013; Newton et al. 2018; Nadler et al. 2019, 2020, 2021) and thus can be used to constrain cosmology through their impact on small-scale clustering statistics (e.g. Benson et al. 2001; Berlind et al. 2003; van den Bosch et al. 2005a; Lange et al. 2019; van den Bosch, Lange \& Zentner 2019). Clearly, accurately modelling the evolution of DM subhalo populations is a prerequisite for their use as a cosmological probe and as a tool to study the particle nature of DM. Unfortunately, since the evolution of DM substructure is highly non-linear, modelling all but the most idealized circumstances has proven analytically intractable. Thus, to date, cosmological $N$-body simulations have been the most common avenue used for studying the demographics of DM substructure.

In recent years, cosmological simulations have successfully and repeatedly passed an important convergence test: as resolution is varied, the SHMFs remain in agreement above the 50-100 particle limit (e.g. Springel et al. 2008; Onions et al. 2012; Knebe et al. 2013; Griffen et al. 2016; van den Bosch \& Jiang 2016; Ludlow, Schaye \& Bower 2019). While this is promising, the physical correctness of cosmological simulations is not guaranteed by the convergence of mass functions alone. Using the state-of-the-art Bolshoi simulation (Klypin, Trujillo-Gomez \& Primack 2011), van den Bosch (2017) found that the evolved SHMF of surviving subhaloes looks identical to the SHMF of disintegrated subhaloes, noting that total subhalo disruption is prevalent. The inferred disruption rates from various studies are extremely high, with roughly 55-65 per cent ( 90 per cent) of subhaloes accreted at $z=1$ (2) being disrupted by the present day (Han et al. 2016; van den Bosch 2017; Jiang \& van den Bosch 2017). Hayashi et al. (2003) have shown that the total binding energy of a halo that is instantaneously stripped down to a sufficiently small radius (encompassing roughly $5-10$ percent of the original mass) can be positive; hence, the authors suggested that such systems could disrupt spontaneously. Motivated by this analysis, subsequent works have incorporated physical disruption via tidal stripping and heating into their models or used such an argument as a justification for their results (Zentner \& Bullock 2003; Taylor \& Babul 2004; Klypin et al. 2015; Garrison-Kimmel et al. 2017). Recently, however, van den Bosch et al. (2018) demonstrated that the boundedness of a subhalo remnant does not depend solely on the total binding energy, but rather on the radial distribution of the binding energies of the constituent particles. In fact, by using idealized simulations with sufficiently high resolution, van den Bosch et al. (2018) showed that it is possible for a self-bound remnant to survive even after 99.9 per cent of the original mass has been stripped. More broadly, the study used analytical arguments to show that neither tidal heating nor tidal stripping alone are capable of causing complete physical disruption of cuspy CDM subhaloes (consistent with earlier work by Peñarrubia et al. 2010). As a follow-up, van den Bosch \& Ogiya (2018) ran a suite of idealized numerical simulations, concluding that disruption of $\mathrm{N}$-body subhaloes in cosmological simulations is largely numerical in nature and can be primarily attributed to (i) discreteness noise caused by insufficient particle resolution and (ii) inadequate softening of gravitational forces (see Mansfield \& Avestruz 2020 for a recent analysis of the impact of the force softening scale on various halo properties). In agreement with these findings, van den Bosch (2017) assessed that $\sim 80$ per cent of subhalo disruption in the Bolshoi simulation is most likely numerical in nature (see Section 2.4 below for details).
If the majority of subhalo disruption in cosmological simulations is indeed numerical, the implications for small-scale cosmology and astrophysics are profound. For example, a disruption-driven reduction in subhalo statistics would result in systematic biases in predictions from subhalo abundance matching (e.g. Conroy, Wechsler \& Kravtsov 2006; Vale \& Ostriker 2006; Guo et al. 2010; Hearin et al. 2013; Chaves-Montero et al. 2016). Semi-analytical models of galaxy and DM substructure evolution (e.g. Taylor \& Babul 2001; Peñarrubia \& Benson 2005; Zentner et al. 2005; Diemand, Kuhlen \& Madau 2007; Kampakoglou \& Benson 2007; Gan et al. 2010; Pullen, Benson \& Moustakas 2014; Jiang \& van den Bosch 2016; Benson 2020; Yang et al. 2020; Jiang et al. 2021) have historically been calibrated to reproduce the results of cosmological simulations and thus end up having inherited any systematic issues present in such simulations. As a specific example, Jiang \& van den Bosch (2016) constructed a semi-analytical model that accurately matches the statistics of subhaloes in the Bolshoi simulation by simply tuning an orbit-averaged mass-loss rate and including an empirical model of subhalo disruption that, by construction, reproduces the disruption demographics in the simulation. As shown in Green \& van den Bosch (2019, hereafter GB19), in the absence of such disruption, the normalization of the evolved SHMF predictions from Jiang \& van den Bosch (2016) is boosted by a factor of 2. Hence, depending on the fraction of subhalo disruption in cosmological simulations that is indeed artificial, it remains possible that such simulations (and derivative semi-analytical models) may be underestimating subhalo abundances by up to a factor of 2 . Such a systematic bias would have serious implications for DM indirect detection searches and could help explain the 'galaxy clustering crisis' in subhalo abundance matching (Campbell et al. 2018), since both of these applications, among others, depend heavily on evolved SHMFs from simulations. As long as the effects of artificial disruption remain as an unknown variable in the analysis of cosmological simulations, we will be unable to extract the maximum amount of cosmological and astrophysical information content that will soon be made available in various large upcoming surveys, including DESI, LSST, EUCLID, and WFIRST. Clearly, there is still work to be done towards better understanding the tidal evolution of DM substructure, hence the motivation of the present study.

Recently, we released SatGen (Jiang et al. 2021), a semianalytical modelling framework for studying galaxy and DM substructure evolution. The core components of the DM-only side of the framework include prescriptions for (i) analytical merger trees (Cole et al. 2000; Parkinson, Cole \& Helly 2008; Benson 2017), from which the internal properties of subhaloes at accretion are derived, (ii) orbital parameter distributions for infalling subhaloes (Zentner et al. 2005; Wetzel 2011; Jiang et al. 2015; Li et al. 2020), (iii) the integration of subhalo orbits, including DF (Chandrasekhar 1943), (iv) the evolved subhalo density profile (ESHDP), which captures how the internal structure of the subhalo responds to tidal heating and stripping (e.g. Hayashi et al. 2003; Peñarrubia et al. 2010; Drakos, Taylor \& Benson 2017; GB19; Errani \& Navarro 2020), and (v) the instantaneous mass-loss rate, which depends on the structure of both the host- and subhalo in addition to the orbit (e.g. Drakos, Taylor \& Benson 2020, this work). In contrast to Jiang \& van den Bosch (2016), which followed van den Bosch, Tormen \& Giocoli (2005b) by only considering orbit-averaged subhalo evolution, SatGen integrates individual subhalo orbits, thereby allowing for a proper treatment of splashback haloes (e.g. Ludlow et al. 2009; Fong \& Han 2021; Diemer 2020, 2021; Aung et al. 2021). As we will show, this treatment of splashback haloes is crucial for properly comparing model predictions with simulation results. 


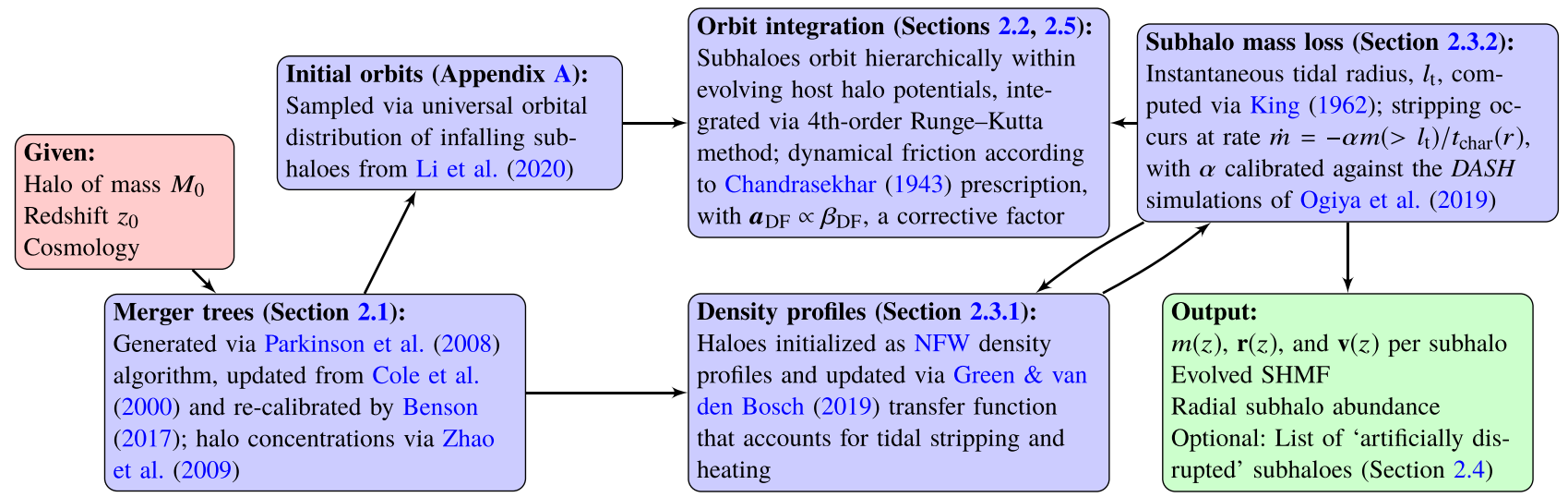

Figure 1. A flowchart that summarizes the SatGen framework employed in this study.

The goal of this work is to build a semi-analytical model of substructure evolution that is independent of any tidal evolution-related numerical artefacts that may be present in cosmological simulations. Thus, in Ogiya et al. (2019), we introduced the Dynamical Aspects of SubHaloes $(D A S H)$ data base, a large library of idealized, highresolution $N$-body simulations of the tidal evolution of individual subhaloes. This simulation library has two key strengths: (i) the simulations span a wide range of parameter space, varying the initial orbital parameters and host- and subhalo concentrations and (ii) the live $N$-body subhaloes satisfy the strict set of convergence criteria laid out in van den Bosch \& Ogiya (2018), suppressing numerical artefacts caused by discreteness noise and inadequate force softening. In GB19, we used DASH to calibrate a highly accurate, simply parametrized empirical model of the ESHDP, which is unimpeded by numerical artefacts and is applicable to a far wider range of subhalo parameter space than that of previous works (Hayashi et al. 2003; Peñarrubia et al. 2010; Drakos et al. 2017). In this work, we use the results of GB19 as a component in a simple, physically motivated model of the instantaneous mass-loss rate. After calibrating this model to faithfully reproduce the subhalo mass trajectories across the range of $D A S H$ simulations, we incorporate it into SatGen, yielding the aforementioned artefact-free semi-analytical model. We use this tool to make predictions for evolved SHMFs, radial profiles, and substructure mass fractions and compare these findings to Bolshoi as an independent attempt to quantify the impact of artificial disruption on the abundance of DM subhaloes in cosmological simulations.

This paper is organized as follows. In Section 2, we describe our methods, giving an overview of SatGen and our modifications, which include the incorporation of the $\mathrm{Li}$ et al. (2020) orbital parameter model (summarized in Appendix A), the ESHDP model of GB19, and an improved, DASH-calibrated mass-loss rate. We also detail our procedures for modelling the impact of artificial disruption and calibrating the DF strength. In Section 3, we present the results of our augmented SatGen model, focusing on SHMFs, radial profiles, substructure mass fractions, and the numerical disruption rate in simulations. We conclude in Section 4 by summarizing our research program, highlighting the updates made to SatGen, and discussing our findings and their implications.

The cosmology used throughout this work is consistent with that of the Bolshoi simulation (Klypin et al. 2011): $\Omega_{\mathrm{m}}=0.270, \Omega_{\Lambda}=$ $0.730, \Omega_{\mathrm{b}}=0.0469, h=0.7, \sigma_{8}=0.82$, and $n_{\mathrm{s}}=0.985$. The halo mass is defined as the mass enclosed within the virial radius, $r_{\mathrm{vir}}$, inside of which the mean density is equal to $\Delta_{\text {vir }}(z)$ times the critical density. For the $\Lambda \mathrm{CDM}$ cosmology adopted in this work, $\Delta_{\text {vir }}(z=0)$ $\approx 100$ and is otherwise well described by the fitting formula presented by Bryan \& Norman (1998). Throughout, we use $m$ and $M$ to denote subhalo and host halo masses, respectively. We use $l$ and $r$ to reference subhalo- and host halo-centric radii, respectively. Projected radii are indicated by upper-case letters. The base- 10 logarithm is denoted by $\log$ and the natural logarithm is denoted by $\ln$.

\section{METHODS}

Our work builds on the original SatGen model that is presented in Jiang et al. (2021); we refer the reader to that paper for any additional model details that are omitted below. In what follows, we highlight the salient features of SatGen and discuss in greater detail the new modifications that we make as part of this study. Fig. 1 presents a schematic flowchart that summarizes all of the individual components of our framework.

\subsection{Merger trees}

Given an input that includes host halo virial mass, $M_{0}$, redshift of observation, $z_{0}$, and underlying cosmology, SatGen generates a user-defined number of halo merger trees that specify the subhalo masses, and redshifts at which they are accreted by the main progenitor of each halo. Merger trees are constructed using the method of Parkinson et al. (2008), which is a modified version of the GALFORM 'binary method with accretion' introduced by Cole et al. (2000). As demonstrated in Jiang \& van den Bosch (2014) and van den Bosch et al. (2014), this method yields results that are in excellent agreement with numerical simulations. ${ }^{1}$ As detailed in Jiang et al. (2021), we use the Parkinson et al. (2008) method with the updated set of parameters advocated for by Benson (2017) that are applicable to the Bryan \& Norman (1998) virial halo mass definition. Each merger tree is characterized by a minimum progenitor mass, $M_{\text {res }}$, which we set to be a fixed fraction, $\psi_{\text {res }}$, of the final host halo mass, i.e. $M_{\text {res }}=$ $\psi_{\text {res }} M_{0}$. The value of $\psi_{\text {res }}$ used varies depending on the application and is specified accordingly. Following Parkinson et al. (2008), the merger tree is sampled using small time-steps of $\Delta z \approx 10^{-3}$; however,

\footnotetext{
${ }^{1}$ As an aside, we acknowledge that several components of the model, including the analytical merger tree algorithm and the orbital parameter distribution model, are still calibrated to agree with cosmological simulations. However, the calibration of these components only depend on properties of unevolved subhaloes (i.e. prior to accretion) and hence are not adversely impacted by any artefacts that may manifest in their subsequent tidal evolution.
} 
in order to reduce memory usage, the tree is subsequently downsampled to a temporal resolution of $\Delta t=\min \left[0.1 t_{\mathrm{dyn}}(z), 0.06 \mathrm{Gyr}\right]$, where $t_{\mathrm{dyn}}(z)=\sqrt{3 \pi /\left[16 G \Delta_{\text {vir }}(z) \rho_{\mathrm{c}}(z)\right]}$ is the redshift-dependent halo dynamical time (see Jiang \& van den Bosch 2016). The maximum time step of $0.06 \mathrm{Gyr}$ is motivated by convergence tests ran during the calibration of our subhalo mass-loss model, which we discuss in Section 2.3.2.

Both host haloes and subhaloes at accretion are assumed to follow a Navarro-Frenk-White density profile (hereafter NFW; Navarro, Frenk \& White 1997) with a concentration parameter, $c_{\mathrm{vir}},{ }^{2}$ that depends on mass and redshift (or time) according to the model introduced by Zhao et al. (2009):

$c_{\mathrm{vir}}\left(M_{\mathrm{vir}}, t\right)=4.0\left[1+\left(\frac{t}{3.75 t_{0.04}}\right)^{8.4}\right]^{1 / 8}$.

Thus, the concentration of the halo at a proper time, $t$, is determined based on the time at which its main progenitor has accumulated a mass of $0.04 M_{\mathrm{vir}}(t)$, denoted $t_{0.04}$. Each branch of the merger tree has its own virial mass accretion history, $M_{\mathrm{vir}}(t)$, that tracks the halo from the time that it attains a mass of $0.04 M_{\text {res }}=0.04 \psi_{\text {res }} M_{0}$ until the time that it merges into a more massive halo. Note that in order to have well-defined concentrations for all progenitor haloes down to a 'leaf mass' of $M_{\text {res }}$, we track the main progenitor branch of each leaf further back in time down to $0.04 M_{\text {res }}$.

SatGen tracks subhaloes of all orders. The main branch, which follows the main progenitor of the $z=z_{0}$ host halo back in time, is considered to be order- 0 . Subhaloes that are directly accreted on to the main host are order-1. These subhaloes themselves can host subsubhaloes, which are order-2, and so on. We use an inclusive mass definition in our merger trees, which means that the summed mass of all order- $k$ subhaloes is included in the mass of their order- $(k-1)$ host. In some of our results (e.g. the SHMFs), we consider subhaloes of all orders; however, due to the inclusive mass definition, we only consider order- 1 subhaloes for other results (e.g. the substructure mass fraction).

\subsection{Orbit integration}

Upon accretion, the initial orbital configuration (i.e. location on the virial sphere, orientation of the orbital plane, and the initial velocity vector) of each subhalo is drawn at random using the state-of-theart universal infall model of Li et al. (2020, see Appendix A for details). Note that this is a significant and important improvement over the approach taken in the original SatGen paper, where it was assumed that all subhaloes initially have an orbital energy of $E_{\text {orb }}=V_{\text {vir,h }}^{2} / 2+\Phi_{\mathrm{h}}\left(r_{\mathrm{vir}}\right)$, where $V_{\mathrm{vir}, \mathrm{h}}$ and $\Phi_{\mathrm{h}}$ are the instantaneous virial velocity and potential of the host halo, and a specific orbital angular momentum of $L_{\text {orb }}=\eta r_{\text {vir }} V_{\text {vir }}$, where $\eta \in[0,1]$ is drawn from a simple sinusoidal probability distribution, $p(\eta)=\pi \sin (\pi \eta) / 2$.

Subhalo orbits are subsequently integrated according to the evolving potential of the immediate host and a simple prescription for DF. In particular, subhaloes are treated as point masses with phase-space coordinates that are updated at each time step by integrating the following equation of motion:

$\ddot{\boldsymbol{r}}=-\nabla \Phi_{\mathrm{h}}+\boldsymbol{a}_{\mathrm{DF}}$.

The integration is performed using a fourth-order Runge-Kutta method. Here, $\boldsymbol{r}$ is the host-centric position vector of the subhalo

${ }^{2}$ We use $c_{\mathrm{vir}, \mathrm{h}}$ and $c_{\mathrm{vir}, \mathrm{s}}$ to refer to host- and subhalo concentrations, respectively. and $\boldsymbol{a}_{\mathrm{DF}}$ is the acceleration due to DF. The latter is modelled using the standard approach of Chandrasekhar (1943), which gives the acceleration as

$\boldsymbol{a}_{\mathrm{DF}}=-4 \pi G^{2} m \ln \Lambda \rho(\boldsymbol{r}) F(<v) \frac{\boldsymbol{v}}{v^{3}}$

(see Mo et al. 2010). Here, $\ln \Lambda=\ln (M / m)$ is the Coulomb logarithm, $M$ and $m$ are the instantaneous masses of the host and subhalo, respectively, $\rho(\boldsymbol{r})$ is the host NFW density profile, $\boldsymbol{v}$ is the relative velocity of the subhalo with respect to the host, and $F(<v)$ is the fraction of local host particles contributing to DF. The velocity distribution of the background particles is assumed to be Maxwellian and isotropic such that

$F\left(<v_{\text {rel }}\right)=\operatorname{erf}(X)-\frac{2 X}{\sqrt{\pi}} \mathrm{e}^{-X^{2}}$.

Here, $X \equiv v_{\text {rel }} /(\sqrt{2} \sigma)$, where $\sigma(\boldsymbol{r})$ is the one-dimensional isotropic velocity dispersion of the host, which we compute using the Jeans equation for hydrostatic equilibrium in a spherical system (e.g. Binney \& Tremaine 2008). We use the orbital velocity of the subhalo for $v_{\text {rel }}$, ignoring the spin of the host halo.

Because of its simplicity and ability to produce results in reasonable agreement with simulations, equation (3) has long been the standard approach for capturing DF in semi-analytical models. However, it is based on a number of assumptions (i.e. a point particle moving in an isotropic, homogeneous background of field particles) that are clearly not justified when modelling the orbital evolution of DM subhaloes. In order to account for these (and other, see Mo et al. 2010) inherent shortcomings, we multiply $\boldsymbol{a}_{\mathrm{DF}}$ by a corrective factor, $\beta_{\mathrm{DF}}$, of order unity. We treat $\beta_{\mathrm{DF}}$ as a free parameter, which allows us to adjust the overall strength of DF (see Section 2.5).

\subsection{Tidal stripping}

As a subhalo orbits its host, it is subjected to tidal stripping and tidal shock heating. As discussed in detail in van den Bosch et al. (2018), neither of these processes can be rigorously treated analytically. Consequently, all previous semi-analytical models of subhalo evolution have calibrated their treatments using cosmological simulations, thereby inheriting any shortcomings present within such simulations (i.e. artificial disruption). The primary goal of this work is to build a semi-analytical model of DM substructure evolution that is calibrated in a way such that its results are not sensitive to such numerical artifacts. We achieve this by calibrating our model against $D A S H$, a large suite of idealized, high-resolution $N$-body simulations that track individual, live $N$-body subhaloes as they orbit a fixed, analytical host halo potential (Ogiya et al. 2019). Both the host halo and the initial $\mathrm{N}$-body subhalo in $D A S H$ are modelled as spherical NFW haloes. $D A S H$ consists of 2,253 simulations spanning a wide range of relevant parameter space, including initial orbital energy and angular momentum, as well as the concentration parameters of both the hostand subhalo. The library consists of various data products generated from each simulation, including the phase-space coordinates of the subhalo centre-of-mass, the subhalo radial density profile, $\rho(l, t)$, and the bound mass fraction, $f_{\text {bound }} \equiv m(t) / m_{\text {acc }}$, where $m_{\text {acc }}$ is the initial subhalo virial mass (i.e. the subhalo mass at accretion), each of which are recorded over 301 snapshots of time. Below, we use these results to calibrate a model that describes the evolution of the density profiles (Section 2.3.1) and bound masses (Section 2.3.2) of subhaloes as they orbit their host (note that the former is required for modelling the latter). 


\subsubsection{The evolved subhalo density profile}

In GB19, we used DASH to calibrate a model that describes how the internal structure of a subhalo evolves in response to tidal stripping and heating. In particular, motivated by the work of Hayashi et al. (2003) and Peñarrubia et al. (2010), GB19 present a 'transfer function' that describes the density profile of a tidally stripped subhalo as a function of its initial density profile and its instantaneous bound mass fraction, $f_{\text {bound }}$. Consequently, the density profile of a subhalo at any time, $t$, is given by

$\rho_{\mathrm{s}}(l, t)=H\left(l \mid f_{\text {bound }}(t), c_{\mathrm{vir}, \mathrm{s}}\right) \rho_{\mathrm{s}}\left(l, t_{\mathrm{acc}}\right)$,

where $c_{\mathrm{vir}, \mathrm{s}}$ is the concentration of the subhalo at accretion and $t_{\mathrm{acc}}$ denotes the time of accretion. The $D A S H$-calibrated transfer function is given by

$H\left(l \mid f_{\text {bound }}, c_{\mathrm{vir}, \mathrm{s}}\right)=\frac{f_{\mathrm{te}}}{1+\left(\frac{l}{l_{\mathrm{s}}}\left[\frac{l_{\mathrm{vir}}-l_{\mathrm{te}}}{l_{\mathrm{vir}} l_{\mathrm{te}}}\right]\right)^{\delta}}$.

Here, $f_{\mathrm{te}}, l_{\mathrm{te}}$, and $\delta$ are all expressed as fitting functions that depend on both $f_{\text {bound }}$ and $c_{\text {vir, s }}$ (see equations $6-8$ and Table 1 of GB19; note that $l_{\mathrm{te}} \equiv r_{\mathrm{te}}$ ), whereas $l_{\mathrm{s}}$ and $l_{\text {vir }}$ are the scale radius and virial radius of the NFW subhalo at accretion. ${ }^{3}$ The transfer function describes how the outer density profile of the subhalo steepens from $\mathrm{d} \ln \rho / \mathrm{d} \ln l=-3$ (i.e. the outer slope of the initial NFW profile) to roughly $-(5-6)$ as the initial subhalo mass is stripped away. In addition, the central density of the subhalo is lowered as $f_{\text {bound }}$ decreases, which is primarily a consequence of revirialization in response to mass-loss.

\subsubsection{Mass-loss rate}

A common approach to modelling the combined impact of tidal stripping and heating (e.g. Taffoni et al. 2003; Zentner \& Bullock 2003; Oguri \& Lee 2004; Zentner et al. 2005; Pullen et al. 2014), which we adopt as well, is to assume that over each time step, $\Delta t$, some portion, $\Delta m$, of the subhalo mass outside of its instantaneous tidal radius, $l_{\mathrm{t}}$, is stripped away. In particular, we set

$\Delta m=-\alpha \frac{\Delta t}{t_{\text {char }}} m\left(>l_{\mathrm{t}}\right)$.

Here, $\alpha$ is a fudge factor that controls the stripping efficiency,

$t_{\text {char }}=\sqrt{\frac{3 \pi}{16 G \bar{\rho}_{\mathrm{h}}(r)}}$

is the characteristic orbital time of the subhalo (identical to the dynamical time introduced in Section 2.1), with $r$ the instantaneous, host-centric radius of the subhalo and $\bar{\rho}_{\mathrm{h}}(r)$ the mean density of the host halo within $r$, and

$l_{\mathrm{t}}=r\left[\frac{m\left(<l_{\mathrm{t}}\right) / M(<r)}{2+\frac{\Omega^{2}(t) r^{3}}{G M(<r)}-\left.\frac{\mathrm{d} \ln M}{\mathrm{~d} \ln r}\right|_{r}}\right]^{1 / 3}$

(King 1962), with $\Omega(t)=|\boldsymbol{r} \times \boldsymbol{v}| / r^{2}$ the instantaneous angular orbital velocity of the subhalo. We have also experimented with other definitions of $t_{\text {char }}$ and $l_{\mathrm{t}}$ but find that this combination, when

\footnotetext{
${ }^{3}$ The dependence on $c_{\mathrm{vir}, \mathrm{s}}$ went unnoticed in Hayashi et al. (2003) and Peñarrubia et al. (2010), both of which only studied subhaloes with a single concentration $\left(c_{\mathrm{vir}, \mathrm{s}}=10\right.$ and 23.1, respectively).
}

used in conjunction with equation (7), is able to reproduce the $D A S H$ results most accurately. 4

We use the $m(t) / m_{\text {acc }}$ trajectories from the $D A S H$ simulations to calibrate $\alpha$ as follows. Given the data products from a particular $D A S H$ simulation, we create interpolators for $r(t), \Omega(t)$, and $m(t) / m_{\text {acc }}$. In order to avoid transient behaviour in the simulations that results from the instantaneous introduction of a subhalo into its host potential (see Ogiya et al. 2019), we initialize our model based on the properties of the DASH subhalo at the beginning of its second orbit (i.e. after it has returned to apocentre for the first time). Given a choice for $\Delta t$ and $\alpha$, we evolve $m(t) / m_{\text {acc }}$ using equation (7), where we set $m\left(>l_{\mathrm{t}}\right)=m(t)-m\left(<l_{\mathrm{t}}\right)$. Here, $m\left(<l_{\mathrm{t}}\right)$ is computed using the ESHDP of equation (5), which depends on the instantaneous value of $m(t) / m_{\text {acc }}$ and the initial $c_{\text {vir, s }},{ }^{5}$ and we demand that $\Delta m \geq 0$ such that the subhalo mass decreases monotonically. For each combination of simulation (indexed by $i$ ) and $\alpha$ value, we compute a cost function, $C(i \mid \alpha)$, which is simply the mean-squared residual in $\log \left[m\left(t_{j}\right) / m_{\text {acc }}\right]$ between our model and DASH averaged over all $n_{\text {apo, } i}$ apocentric passages subsequent to the initialization of our model (indexed by $j$ ). We then determine the total cost for a given $\alpha$ by computing the mean of the $C(i \mid \alpha)$ taken over all of the DASH simulations, which can be written explicitly as

$C(\alpha)=\sum_{i}^{n_{\text {sim }}} \frac{C(i \mid \alpha)}{n_{\text {sim }}}=\sum_{i}^{n_{\text {sim }}} \sum_{j}^{n_{\text {apo }, i}} \frac{\log ^{2}\left[m_{\text {model }, i}\left(t_{j}\right) / m_{\mathrm{DASH}, i}\left(t_{j}\right)\right]}{n_{\text {sim }} n_{\text {apo }, i}}$.

We emphasize that this cost function weighs each simulation equally, which is motivated by the fact that $D A S H$ samples the parameter space of orbits and halo concentrations according to a cosmological simulation-inferred joint probability distribution. The cost function depends somewhat on the time step used to integrate the model predictions (see equation 7), but we find that the results converge with $\Delta t=0.06 \mathrm{Gyr}$, which we adopt throughout as the maximum time step for integrating the evolution of the subhalo in SatGen.

We find that $C(\alpha)$ is minimized for $\alpha \simeq 0.6$, for which the rootmean-square error in the apocentric mass predictions is $0.097 \mathrm{dex}$. In order to look for any secondary parametric dependence that the optimal $\alpha$ may have, we determine the best-fitting $\alpha$ on a persimulation basis, which we denote $\alpha_{i}$. We then look at the correlation between $\alpha_{i}$ and the concentrations of the host- and subhalo as well as with the orbital parameters. We find that $\alpha_{i}$ depends strongly on $c_{\mathrm{vir}, \mathrm{s}} / c_{\mathrm{vir}, \mathrm{h}}$. By binning the simulations by $c_{\mathrm{vir}, \mathrm{s}} / c_{\mathrm{vir}, \mathrm{h}}$ and taking the median $\alpha_{i}$ in each bin, we find a power-law relation that is well fit by

$\alpha=0.55\left(\frac{c_{\mathrm{vir}, \mathrm{s}} / c_{\mathrm{vir}, \mathrm{h}}}{2}\right)^{-1 / 3}$.

This relation captures the fact that subhaloes that are more compact relative to their host are more resilient to stripping. We adopt this parametrization of $\alpha$ in SatGen, emphasizing that, for typical values of $c_{\mathrm{vir}, \mathrm{s}} / c_{\mathrm{vir}, \mathrm{h}}$, the concentration-dependence has a $\lesssim 30$ per cent effect. In determining $\alpha$, we use the instantaneous host $c_{\mathrm{vir}, \mathrm{h}}$ (which evolves as long as the host itself has not yet become a subhalo) whereas the subhalo $c_{\mathrm{vir}, \mathrm{s}}$ is fixed to its value at infall.

${ }^{4}$ The tidal radius is only an approximation of the zero-velocity surface, which itself is neither spherical nor infinitesimally thin, and different authors often adopt different definitions. See Read et al. (2006), Tollet et al. (2017), and van den Bosch et al. (2018) for detailed discussions.

${ }^{5}$ This enclosed mass profile is not analytical. Hence, in SatGen, we provide an interpolator for $m(<l) / m_{\text {acc }}$ (and $\sigma(l)$, the one-dimensional isotropic velocity dispersion), which is itself a function of $l, m(t) / m_{\mathrm{acc}}$, and $c_{\mathrm{vir}, \mathrm{s}}$. We interpolate over $\log \left[m(t) / m_{\text {acc }}\right]$ and $\log \left(c_{\mathrm{vir}, \mathrm{s}}\right)$ using cubic B-splines and patch the surfaces together in $\log (l)$-space linearly. 


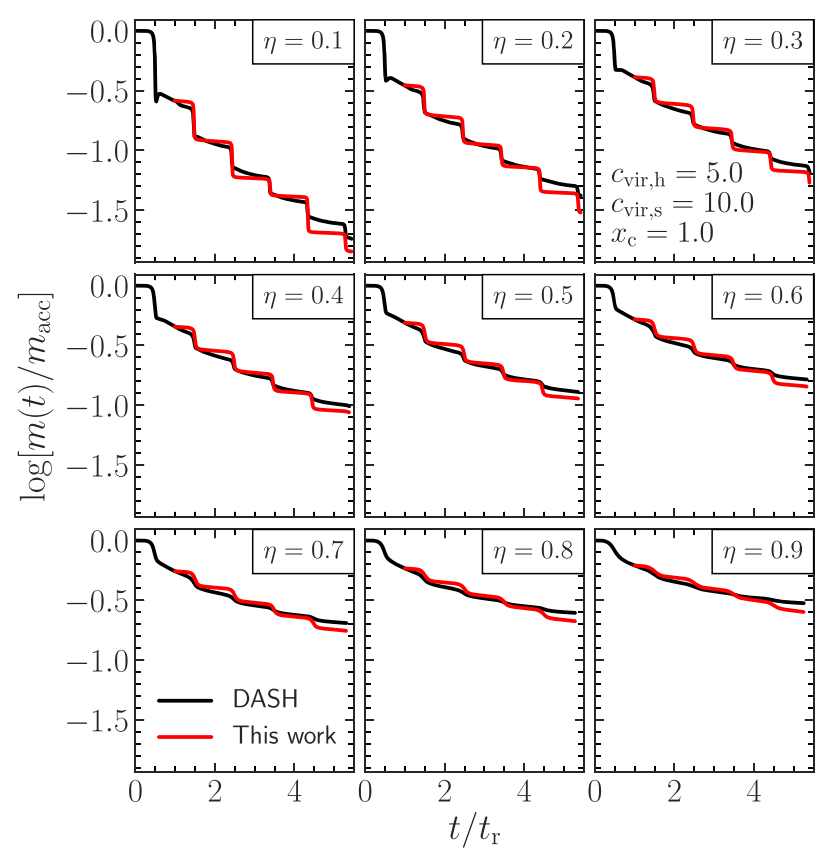

Figure 2. A comparison between our calibrated mass-loss model predictions and the DASH $m(t) / m_{\text {acc }}$ trajectories of several simulations. The times are normalized by the radial orbital period, $t_{\mathrm{r}}$. We fix $c_{\mathrm{vir}, \mathrm{h}}=5, c_{\mathrm{vir}, \mathrm{s}}=10$, and $x_{\mathrm{c}}=1$ (these parameters are typical of systems seen in cosmological simulations; see e.g. Ogiya et al. 2019), demonstrating that the model performance is strong over a wide range of circularity values, ranging from highly elliptical $(\eta=0.1)$ to nearly circular orbits $(\eta=0.9)$.

Although it is tempting to compare our best-fitting value for $\alpha$ to that of previous semi-analytical models that rely on equation (7), such a comparison is frustrated by the fact that different studies have used different forms for $t_{\text {char }}$ and/or $l_{\mathrm{t}}$ (see van den Bosch et al. 2018; Drakos et al. 2020 for detailed discussions). In addition, none of the previous studies have accounted for the detailed evolution of the subhalo density profile (as in, e.g. equation 5), rendering such a comparison moot. We do emphasize, though, that by calibrating our model to the idealized $D A S H$ simulations, rather than to cosmological simulations, such as in Zentner et al. (2005) and Pullen et al. (2014), our calibration is not adversely impacted by potential issues resulting from artificial disruption.

Fig. 2 compares the $m(t) / m_{\text {acc }}$ trajectories of several $D A S H$ simulations (black lines) to predictions based on our mass-loss model (red lines). In each case, $c_{\mathrm{vir}, \mathrm{h}}=5, c_{\mathrm{vir}, \mathrm{s}}=10$, and the orbital energy, $E$, is that of a circular orbit at the virial radius of the host (i.e. $x_{\mathrm{c}}$ $\equiv r_{\mathrm{c}}(E) / r_{\mathrm{vir}}=1$, where $r_{\mathrm{c}}(E)$ is the radius of a circular orbit with energy $E$ ). Different panels correspond to different values of the orbital circularity, $\eta \equiv L / L_{\mathrm{c}}(E)$, as indicated, where $L$ is the orbital angular momentum and $L_{\mathrm{c}}(E)$ is the angular momentum of a circular orbit with the same orbital energy as that of the subhalo. Clearly, our model tracks the $D A S H m(t) / m_{\text {acc }}$ curves quite faithfully over $\sim 5$ radial orbital periods. Importantly, the performance of the model is strong over the full range of $\eta$, spanning from orbits that are close to radial $(\eta=0.1)$ to those that are close to circular $(\eta=$ 0.9 ), a feat that has proven difficult for previous semi-analytical models of subhalo mass evolution (cf. Peñarrubia et al. 2010; Drakos et al. 2020). Although not shown, we emphasize that the model performs comparably for other configurations as well. In particular, the concentration dependence built into the parametrization of the

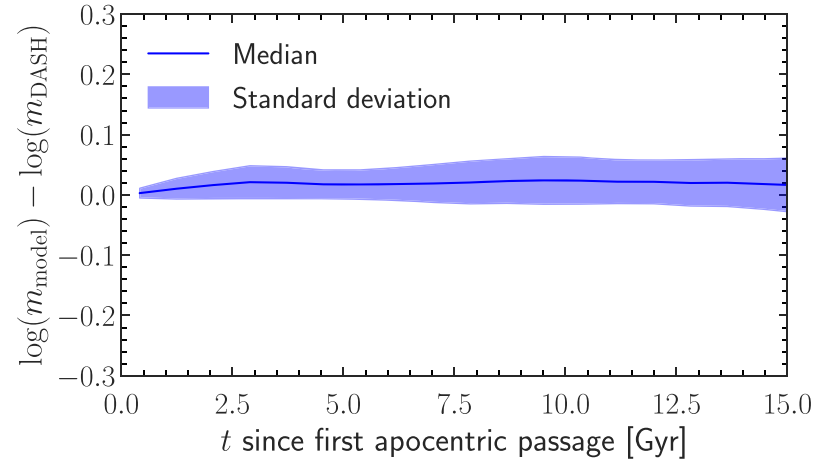

Figure 3. The time evolution of the median and standard deviation of the log-residuals between our mass-loss model predictions and the simulated mass trajectories taken over the ensemble of DASH simulations. The model performs well over the full parameter space, with minimal bias and scatter for longer than a Hubble time.

stripping efficiency (i.e. equation 11) considerably improves the predictions made for systems with $c_{\mathrm{vir}, \mathrm{s}} / c_{\mathrm{vir}, \mathrm{h}}$ ratios that deviate significantly from two.

We use the mass-loss model to predict the mass evolution of every simulated DASH subhalo. In Fig. 3, we plot the time evolution of the median and standard deviation of the log-residuals between our model predictions and the DASH mass trajectories. We find that the mass-loss model performs well over the full parameter space, with minimal bias and scatter for longer than a Hubble time. After $15 \mathrm{Gyr}$ of evolution, the scatter in the log-residuals of our mass-loss model reaches only $0.04 \mathrm{dex}$; hence, the impact of mass evolution error will be subdominant to the intrinsic halo-to-halo variance in our quantities of interest.

\subsubsection{Stripping of higher order substructure}

In addition to the treatment of subhalo mass-loss, SatGen also implements a procedure for the splashback release of higher order subhaloes. Specifically, each time step that an order- $k$ subhalo lies outside of the tidal radius of its order- $(k-1)$ host, it has a probability of min $\left[\alpha \Delta t / t_{\text {char }}(r), 1\right]$ of being released from its host and becoming an order- $(k-1)$ subhalo. Here, $\alpha$ and $t_{\text {char }}(r)$ are computed for the order- $(k-1)$ host with respect to its order- $(k-2)$ parent, which is responsible for stripping off the order- $k$ subhalo. In the event of release, the phase-space coordinates of the subhalo with respect to its new, order- $(k-2)$ host are the superposition of its original coordinates with respect to its old, order- $(k-1)$ host and those of the old host with respect to the order- $(k-2)$ system. The remaining bound mass of the original, order- $k$ subhalo is instantaneously removed from the mass of its old, order- $(k-1)$ parent in order to enforce mass conservation.

\subsubsection{Resolution limits}

As discussed in Section 2.1, SatGen has a merger tree resolution limit, which sets the smallest subhalo mass at accretion to $\psi_{\text {res }} M_{0}$. Such a limit is necessary in order to maintain computational feasibility, as the size of the merger tree grows exponentially with decreasing $\psi_{\text {res }}$. However, once accreted, a subhalo is evolved in SatGen for as long as its mass $m \geq \phi_{\text {res }} m_{\text {acc }}$. Here, $\phi_{\text {res }}$ is the imposed resolution limit for the bound mass fraction. Our default is to set $\phi_{\text {res }}=\psi_{\text {res }}$, which ensures that the least (most) massive subhaloes are tracked 
down to $m=\phi_{\text {res }} \psi_{\text {res }} M_{0}$ ( $\left.m=\psi_{\text {res }} M_{0}\right)$. In what follows, both resolution limits are adjusted depending on the specific topic that is under investigation.

\subsection{Artificial disruption}

Recently, van den Bosch et al. (2018) and van den Bosch \& Ogiya (2018) carried out a comprehensive analytical and numerical study focused on subhalo disruption. Using simple, physical arguments, the authors demonstrate that the inner remnant of an NFW subhalo should survive even when tidal shock heating has injected an amount of energy that is many multiples of the binding energy of the subhalo and/or tidal stripping has removed more than 99.9 percent of the initial subhalo mass. This claim is confirmed using idealized $\mathrm{N}$ body simulations of subhalo evolution (similar to $D A S H$ ), with the authors concluding that the majority of subhalo disruption seen in cosmological simulations is numerical in nature.

Let us use Bolshoi as our example cosmological simulation for considering the rate of artificial disruption. van den Bosch (2017) used the merger trees from Bolshoi to separate subhalo evolution into several unique channels. Of these channels, the disruption (D) and withering (W) branches pertain specifically to numerical subhalo disruption. A subhalo in one snapshot that evolves along the D channel has no descendent at any subsequent snapshot. On the other hand, a subhalo that evolves along the $\mathrm{W}$ channel has a descendent in the subsequent snapshot that falls below the 50 particle resolution limit imposed by the author. By studying these branches, van den Bosch (2017) concludes that artificial disruption (D) occurs at a rate of 2.4 per cent/Gyr and falling below the mass limit (W) occurs at a rate of $\sim 10$ per cent/Gyr. When combined, the total numerical disruption $(\mathrm{W}+\mathrm{D})$ rate in Bolshoi is roughly 13 per cent/Gyr, resulting in $\sim 65$ per cent of subhaloes accreted at $z=1$ being numerically disrupted by the present day, in good agreement with independent estimates made by Han et al. (2016) and Jiang \& van den Bosch (2017). As long as simulations have a finite number of particles, the $\mathrm{W}$ channel will exist. However, its significance diminishes as simulation resolution limits move toward smaller halo masses that are below all scales of interest. The D channel, on the other hand, is more alarming, since it represents subhaloes, often well above the mass limit, that simply disappear from the merger tree. The 2.4 per cent/Gyr of the D channel translates to roughly 20 per cent of subhaloes accreted at $z=1$ being (artificially) disrupted by $z=0$.

In order to assess the overall significance of numerical disruption, we aim to model both the impact of the $\mathrm{W}$ branch in isolation as well as the impact of both the $\mathrm{W}$ and $\mathrm{D}$ channels in combination on the SatGen results. As introduced in Section 2.3.2, the W branch subhaloes in SatGen are simply those with a final mass that has fallen below the merger tree resolution limit, $\psi_{\text {res }} M_{0}$. Although even SatGen has an imposed resolution limit on how far down in $\mathrm{m} / \mathrm{m}_{\text {acc }}$ it tracks a subhalo, we can nevertheless make reasonable predictions in the absence of withering by considering all subhaloes with $\mathrm{m} / \mathrm{m}_{\text {acc }}$ $\geq \phi_{\text {res }}=10^{-5}$, which we refer to as the 'wither-free' fiducial model. Whenever withering is considered, the subhalo mass limit is set to $\psi_{\text {res }} M_{0}$ instead.

A key goal of this work is to assess the impact of artificial disruption on the subhalo demographics in cosmological simulations. We are able to do so by adding a model of artificial disruption into SatGen and adjusting its strength (if needed) such that the SatGen predictions (which are inherently free of artificial disruption) reproduce the abundance of subhaloes in a simulation such as Bolshoi. This feat also requires properly accounting for the mass resolution limit (withering) of the simulation of interest. We implement a version of

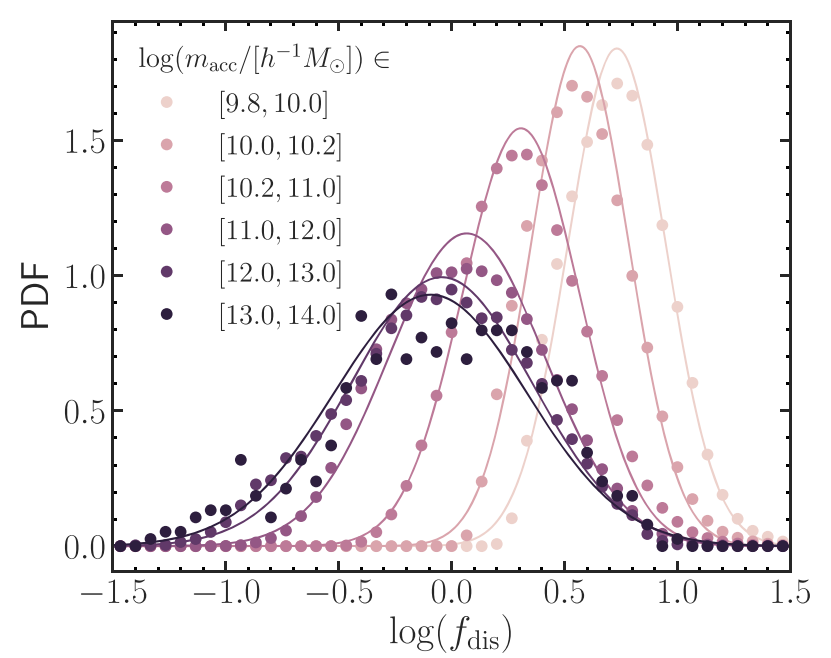

Figure 4. The $\log \left(f_{\text {dis }}\right)$ distribution of disrupted Bolshoi subhaloes. Here, $f_{\text {dis }}$ is a proxy for the mass below which a particular subhalo is artificially disrupted in the simulation (see equation 12). Each colour denotes a different $m_{\text {acc }}$ bin. The points are calculated using all Bolshoi subhaloes that disrupt at $z \geq 0.0148$. The solid curves correspond to our model that is fit to the Bolshoi disruption data (equation [13]). The $f_{\text {dis }}$ are distributed lognormal, with $\mu$ decreasing (and $\sigma$ increasing) as $m_{\text {acc }}$ is increased (up to a saturation point, above which the distribution remains fixed).

the artificial disruption mechanism used in Jiang \& van den Bosch (2016), which itself is based on the prescription of Taylor \& Babul (2004). A subhalo is marked as artificially disrupted when its mass, $m(t)$, falls below its 'disruption mass', given by

$m_{\mathrm{dis}}=m_{\mathrm{acc}}\left(<f_{\mathrm{dis}} l_{\mathrm{s}}\right)=m_{\mathrm{acc}} \frac{f\left(f_{\mathrm{dis}}\right)}{f\left(c_{\mathrm{vir}, \mathrm{s}}\right)}$.

Here, $m_{\text {acc }}(<l)$ denotes the enclosed NFW mass profile of the subhalo at accretion, and $f(x)=\ln (1+x)-x /(1+x)$. The sensitivity of haloes to artificial disruption is set by $f_{\text {dis }}$, which represents the effective radius that a halo can be stripped down to before being disrupted. Under this prescription, haloes with a larger initial concentration are more resilient to disruption. This approach to modelling (artificial) disruption has been employed in previous semi-analytical models (e.g. Hayashi et al. 2003; Taylor \& Babul 2004; Zentner et al. 2005), with $f_{\text {dis }}$ ranging from 0.1 to 2.0 .

Rather than select a fixed value for $f_{\text {dis }}$, Jiang \& van den Bosch (2016) randomly sampled $f_{\text {dis }}$ for each subhalo from a universal lognormal distribution. We augment this approach by calibrating a more general model of $f_{\text {dis }}$ that takes into account a dependence on $m_{\text {acc }}$ that we identify in the Bolshoi subhaloes. Using all halo catalogues from Bolshoi ${ }^{6}$ with $z \geq 0.0148,{ }^{7}$ we extract $m_{\text {acc }}, c_{\text {vir, }}$, and $m_{\text {dis }}$ from all D channel subhaloes from which the $f_{\text {dis }}$ of each corresponding subhalo is calculated. We find that the distribution of $f_{\text {dis }}$ has minimal dependence on redshift and host halo mass, but has a strong dependence on $m_{\text {acc }}$. As shown in Fig. 4, when binned by $m_{\text {acc }}$, the $f_{\text {dis }}$ distribution is roughly lognormal with a log-mean, $\mu$, and log-variance, $\sigma^{2}$, that increases and decreases, respectively, with decreasing $m_{\text {acc }}$. This indicates that subhaloes that are more massive at accretion are less

\footnotetext{
${ }^{6}$ Available at http://www.slac.stanford.edu/ behroozi/Bolshoi_Catalogs/.

${ }^{7} \mathrm{We}$ omit using the several snapshots closer to $z=0$ in order to avoid contaminating the $\mathrm{D}$ branch with instances of snapshot-limited failed phantom patching (see discussion in van den Bosch 2017).
} 
likely to undergo artificial disruption. However, note that this trend in $m_{\text {acc }}$-space appears to saturate at the massive end. Motivated by these findings, we model $f_{\text {dis }}\left(m_{\text {acc }}\right)$ as a lognormal with

$\mu=A+B\left[1+\left(\log \left(m_{\mathrm{acc}}\right)+C\right)^{-2}\right]^{-1 / 2}$, and

$\sigma=D+E \mu+F \mu^{2}$.

Using maximum-likelihood estimation, we obtain the best-fitting parameters of $(A, B, C, D, E, F)=(3.08,-3.26,-8.89,0.38$, $-0.51,0.40)$. The corresponding best-fitting model is indicated as solid lines in Fig. 4 and captures all of the salient details of the data.

When modelling artificial disruption in SatGen, we randomly draw a value of $f_{\text {dis }}$ from the lognormal distribution described by equation (13) for each subhalo at accretion. Subsequently, the subhalo is marked as artificially disrupted once its mass drops below its assigned $m_{\mathrm{dis}}$, which is computed using equation (12). By applying this artificial disruption mechanism, SatGen is able to faithfully reproduce the statistics of the Bolshoi D branch subhaloes. We caution that this particular treatment of artificial disruption is only applicable to Bolshoi. Readers interested in modelling artificial disruption in another simulation must first characterize the corresponding $f_{\text {dis }}$ statistics of the particular simulation.

\subsection{Dynamical friction strength}

In order to calibrate the overall efficiency of DF, which we quantify through the correction factor, $\beta_{\mathrm{DF}}$, we seek a measurement made from cosmological simulations that is both sensitive to DF and insensitive to any underlying artificial disruption. In van den Bosch et al. (2016), the authors study the segregation of subhaloes in Bolshoi. They measure the mean host-centric radius of subhaloes, $\left\langle r / r_{\mathrm{vir}}\right\rangle$, as a function of their redshift of accretion, $z_{\text {acc }}$.

Plotting $\left\langle r / r_{\mathrm{vir}}\right\rangle$ (averaged over thousands of subhaloes) as a function of $z_{\text {acc }}$ (see Fig. 7 in van den Bosch et al. 2016) reveals the characteristics of an orbit (for $z_{\text {acc }} \lesssim 0.5$ ). Subhaloes accreted at $z_{\text {acc }} \sim 0.1$ have just reached pericentre for the first time, while those that are at their first apocentric passage since accretion typically were accreted around $z_{\text {acc }} \sim 0.25$. Note that phase mixing, which is primarily driven by variance in the orbital periods of subhaloes at infall, ${ }^{8}$ results in a lack of orbital coherence for subhaloes accreted before $z_{\text {acc }} \sim 0.5$; this is made apparent by the lack of clear apo- or pericentric passages in $\left\langle r / r_{\mathrm{vir}}\right\rangle$ at high $z_{\text {acc }}$. Interestingly, the $\left\langle r / r_{\text {vir }}\right\rangle\left(z_{\text {acc }}\right)$ curves show a clear dependence on $m_{\text {acc }} / M_{0}$. In particular, subhaloes with larger $m_{\text {acc }} / M_{0}$ reach a smaller apocentric $\left\langle r / r_{\text {vir }}\right\rangle$ at $z_{\text {acc }} \sim 0.25$ than their less-massive counterparts (see Fig. 10 in van den Bosch et al. 2016, which is reproduced as the dashed lines in Fig. 5). This is a manifestation of DF, which allows us to calibrate $\beta_{\text {DF }}$ as follows. ${ }^{9}$

We construct a set of $\sim 45000$ merger trees (with $\psi_{\text {res }}=10^{-3}$ ) with host masses consistent with the $\sim 9000$ host halo sample used in van den Bosch et al. (2016) - we augment our sample by generating five trees per unique host mass. We evolve the subhaloes with SatGen, repeating the procedure for several values of $\beta_{\mathrm{DF}}$ covering the range $[0,1.5]$. We apply the same selection function as used in van den

${ }^{8}$ The efficiency of phase mixing is further enhanced by DF, which impacts the subhalo orbit differently depending on $m_{\text {acc }}$, and variance in the host mass accretion history, which itself affects the evolution of the subhalo orbit between infall and the present day.

${ }^{9}$ Since artificial disruption is rare for subhaloes that were only accreted recently, this feature is not significantly impacted by artificial disruption.

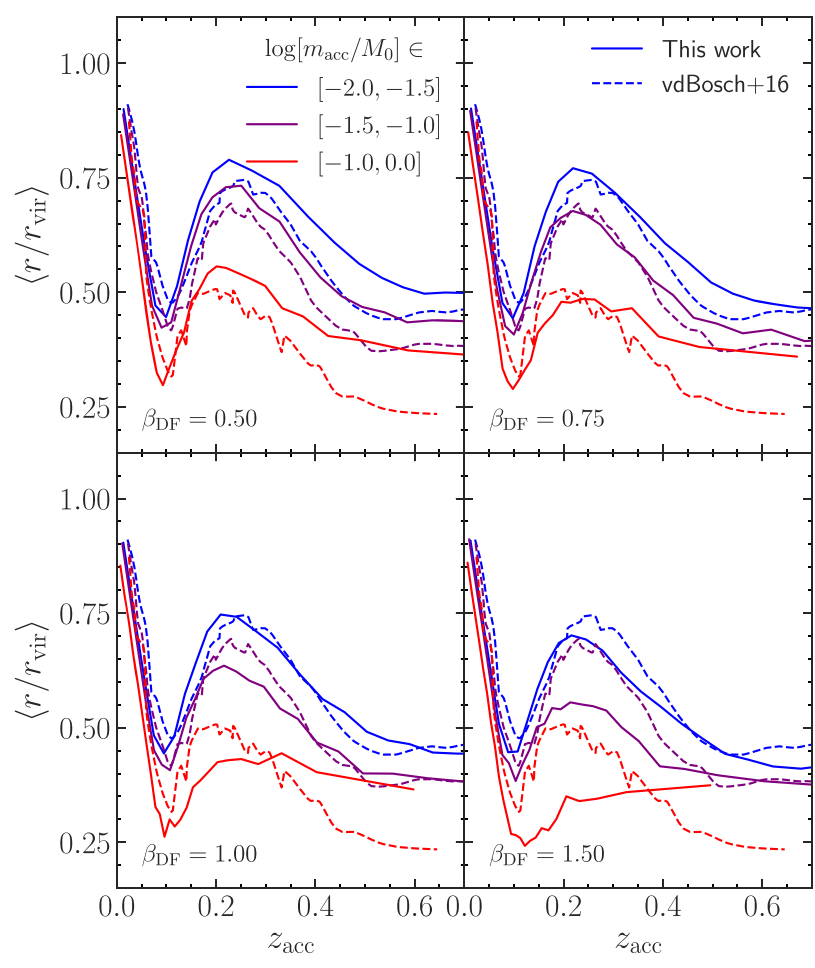

Figure 5. A comparison between the $\left\langle r / r_{\text {vir }}\right\rangle-z_{\text {acc }}$ relation of Bolshoi subhaloes binned by infall mass relative to $z=0$ host mass, $m_{\text {acc }} / M_{0}$ (dashed curves, reproduced from Fig. 10 in van den Bosch et al. 2016), and analogous predictions by SatGen (solid curves). Each panel corresponds to a different value of $\beta_{\mathrm{DF}}$ (indicated in the bottom left-hand of each panel), which controls the strength of DF (see Section 2.2). We adopt $\beta_{\mathrm{DF}}=0.75$ as the fiducial value used in SatGen, since this yields the best agreement with respect to the peak values of $\left\langle r / r_{\text {vir }}\right\rangle$ at $z_{\text {acc }} \sim 0.25$, which corresponds to the first apocentric passage since infall.

Bosch et al. (2016): we only consider subhaloes with $m(z=0) / M_{0}$ $\geq 10^{-3}, m_{\text {acc }} / M_{0} \geq 10^{-2}$, and $m(z=0) / m_{\text {acc }} \geq 10^{-1}$. We first bin the subhaloes by $m_{\text {acc }} / M_{0}$ and then compute $\left\langle r / r_{\text {vir }}\right\rangle$ in $z_{\text {acc }}$ bins, which are chosen such that the number of subhaloes in each bin is the same.

Fig. 5 shows the resulting $\left\langle r / r_{\text {vir }}\right\rangle-z_{\text {acc }}$ relation for four values of $\beta_{\mathrm{DF}}$ as indicated. Clearly, when $\beta_{\mathrm{DF}}=0.75$, SatGen is able to very closely reproduce the simulation results. For $\beta_{\mathrm{DF}}=0.5$ (1.0), Sat Gen yields apocentric $\left\langle r / r_{\text {vir }}\right\rangle$ that are too large (small) relative to Bolshoi, with the disagreement being more significant for the subhaloes with larger $m_{\text {acc }} / M_{0}$ that are more strongly influenced by DF. These findings are independent of whether or not we incorporate artificial disruption using the method described in Section 2.4, which is consistent with the notion that the $\left\langle r / r_{\text {vir }}\right\rangle-z_{\text {acc }}$ relation should be relatively insensitive to artificial disruption (at least for $z_{\text {acc }} \lesssim 0.5$ ). Hence, in what follows, we adopt $\beta_{\mathrm{DF}}=0.75$ as our fiducial DF strength. In Section 3.3 , we quantify the impact of $\beta_{\mathrm{DF}}$ on our substructure mass fraction predictions (by comparing to the 'natural' case of $\beta_{\mathrm{DF}}=1$ ), demonstrating that our results are insensitive to its exact value.

\section{RESULTS}

Given a host halo mass, $M_{0}$, target redshift, $z_{0}$, and requested number of individual trees, $N_{\text {tree }}$, SatGen produces $N_{\text {tree }}$ subhalo catalogues at each redshift time step until $z_{0}$. These catalogues trace the mass and phase-space coordinates of each subhalo over its evolution. In this section, we present the results of these SatGen subhalo catalogues 
and make comparisons to Bolshoi. We begin by studying SHMFs (and subhalo maximum circular velocity functions), comparing SatGen results with and without the artificial disruption mechanism and discuss the significant impact of splashback subhaloes (Section 3.1). In Section 3.2, we proceed to incorporate position data by calculating the radial profile and the (projected) enclosed substructure mass fraction, $F_{\text {sub }}(<R)$. In Section 3.3, we quantify how $f_{\text {sub }}\left(<r_{\text {vir }}\right)$ varies with both $M_{0}$ and resolution limit, $\psi_{\text {res }}$. We also quantify the impact of model parameters (i.e. stripping efficiency and DF strength) on $f_{\text {sub }}$ predictions. Lastly, in Section 3.4, we estimate the total rate of numerical disruption that occurs via the $\mathrm{W}$ and $\mathrm{D}$ channels modelled by SatGen, which we compare to the numerical disruption rate of Bolshoi haloes (as measured by van den Bosch 2017).

\subsection{Subhalo mass/velocity functions}

We turn our attention to the SatGen predictions of the SHMFs for a $10^{14.2} h^{-1} \mathrm{M}_{\odot}$ host. In a cosmological simulation, the SHMF, $\mathrm{d} N / \mathrm{d} \log \left(m / M_{0}\right)$, is calculated using subhaloes of all orders enclosed within the virial radius of the host. Note that since we use an inclusive mass definition and consider all orders of substructure, the total substructure mass is not the mass-weighted integral of the SHMF. The left-hand panel of Fig. 6 shows the mean SHMF computed from 10000 trees (with $\psi_{\text {res }}=10^{-4}$ ). For comparison, the filled symbols indicate the mean SHMF of the 282 Bolshoi host haloes with $\log \left(M_{0} /\left[h^{-1} \mathrm{M}_{\odot}\right]\right) \in[14.0,14.5]$ (with a mean of 14.2). On the high- $m / M_{0}$ end, the Bolshoi SHMF is somewhat noisy due to limited halo statistics. However, a comparison at the low- $m / M_{0}$ end illustrates that SatGen predicts a $\sim 0.1 \mathrm{dex}$ enhancement in the SHMF relative to Bolshoi.

If the primary cause of the disagreement between the SatGen and Bolshoi SHMFs is artificial disruption, then the application of our artificial disruption mechanism (Section 2.4) should result in better agreement between the model and simulation results. Indeed, Fig. 6 shows that 'turning on' D channel disruption suppresses the SatGen SHMF by $\sim 0.05-0.1$ dex at low $m / M_{0}$, bringing it into closer agreement with Bolshoi. Restricting to $-3.9 \leq \log \left(m / M_{0}\right) \leq-0.8$, we fit a power law to the SHMF of the form $\mathrm{d} N / \mathrm{d} \log \left(m / M_{0}\right) \propto A\left(m / M_{0}\right)^{B}$. For the fiducial SatGen results, we find $A=-1.066$ and $B=$ -0.885 , whereas the disruption mechanism slightly suppresses both the normalization and the magnitude of the slope, resulting in $A=$ -1.085 and $B=-0.868 .{ }^{10}$ The reduced slope is a consequence of the $m_{\text {acc }}$-dependence of our artificial disruption model. Note that the Bolshoi SHMF is too noisy to compute a reliable estimate of the slope over the same mass range, but it agrees well visually with the SatGen 'disruption on' results.

Thus far, these results suggest that artificial disruption has, at most, an $\sim 20$ per cent impact on the SHMF of well-resolved host haloes, with the difference being strongest at low $m / M_{0}$. We discuss more quantitatively the impact of disruption and its dependence on halo mass relative to the simulation resolution limit in Section 3.3, which focuses on the substructure mass fraction. The modest decrease in the SHMF normalization due to disruption predicted by SatGen is considerably smaller than the factor of 2 suppression suggested by the GB19-interpretation of the Jiang \& van den Bosch (2016) model. This is because their orbit-averaged model did not take into account the impact of splashback haloes, which are subhaloes that

\footnotetext{
${ }^{10}$ These SHMF slopes are consistent with previous work, which typically find $-1.0 \lesssim B \lesssim-0.8$ (e.g. Boylan-Kolchin et al. 2010; Gao et al. 2012; van den Bosch \& Jiang 2016).
}

have previously fallen within the host $r_{\text {vir }}$ (thus becoming included in the halo merger tree) but instantaneously lie outside of $r_{\mathrm{vir}}$ at $z=0$ (and therefore are typically not included in simulationbased measurements of the SHMF). Benson (2017) briefly discusses this limitation of standard EPS-based approaches to substructure modelling, concluding that a full dynamical model (such as SatGen) is necessary in order to properly account for splashback haloes. In Fig. 6, we illustrate that when splashback haloes are included in the SHMF, the subhalo abundance is enhanced by $\sim 0.2-0.25$ dex on the low- $m / M_{0}$ end relative to the fiducial model. When the 'fiducial + splashback' curve is compared directly to Bolshoi, we find the same $\sim 0.3$ dex (factor of 2) difference as GB19. This highlights the importance of properly accounting for splashback haloes by integrating subhalo orbits. Consistent with these predictions, Bakels et al. (2021) recently reported that roughly half of all subhaloes lie outside of $1.2 r_{200 \mathrm{c}}\left(\sim r_{\text {vir }}\right)$ in a sample of galaxy- to group-mass host haloes studied in a cosmological simulation (consistent with previous work by, e.g. Gill, Knebe \& Gibson 2004; Ludlow et al. 2009).

In addition to mass, another property of subhaloes that is often used (especially in subhalo abundance matching, e.g. Trujillo-Gomez et al. 2011; Hearin et al. 2013; Reddick et al. 2013; Zentner, Hearin \& van den Bosch 2014) is its maximum circular velocity, $V_{\max }$. Hence, we also present results for the subhalo maximum circular velocity function (SHVF), $\mathrm{d} N / \mathrm{d} \ln \left(V_{\mathrm{max}} / V_{\mathrm{vir}, \mathrm{h}}\right)$, where $V_{\mathrm{vir}, \mathrm{h}}$ denotes the virial velocity of the host halo at $z=0$. Since the enclosed mass profile corresponding to the GB19 ESHDP is not analytical, we compute $V_{\max }$ by multiplying the subhalo's maximum circular velocity at accretion,

$V_{\text {max }, \mathrm{acc}}=\sqrt{\frac{G m_{\mathrm{acc}}}{l_{\mathrm{vir}}} \times \frac{0.216 c_{\mathrm{vir}, \mathrm{s}}}{f\left(c_{\mathrm{vir}, \mathrm{s}}\right)}}$

(Bullock et al. 2001), by the 'tidal track' (Peñarrubia, Navarro \& McConnachie 2008) expression for $V_{\max } / V_{\max }$ acc, given by equation (11) in GB19. This tidal track itself is a function of both $\mathrm{m} / \mathrm{m}_{\text {acc }}$ and $c_{\mathrm{vir}, \mathrm{s}}$, as given by equations (12) and (13) in GB19.

Using the same 10000 trees as those used to compute the SHMF, we obtain the SHVF shown in the right-hand panel of Fig. 6. Just as for the SHMF, the filled symbols indicate the corresponding result computed from the 282 Bolshoi host haloes with $\log \left(M_{0} /\left[h^{-1} \mathrm{M}_{\odot}\right]\right) \in[14.0,14.5]$. As is evident, the abundance of subhaloes with $\log \left(V_{\max } / V_{\text {vir,h }}\right) \lesssim-0.4$ predicted by SatGen is about 0.15 dex higher than that of Bolshoi. However, when including artificial disruption, the SatGen predictions once again agree closely with the simulation results.

\subsection{Radial profiles}

Having looked at the subhalo mass and velocity functions, we proceed to incorporate additional spatial information by considering several other quantities of interest. First, we measure the subhalo radial distribution, $\mathrm{d} \tilde{N} /\left.\mathrm{d} x^{3}\right|_{\text {sub }}$, as the number of subhaloes per unit shell volume as a function of $x=r / r_{\text {vir }}$, which we normalize to unity at $r_{\text {vir }}$. We assess the radial bias of the subhaloes by comparing $\mathrm{d} \tilde{N} /\left.\mathrm{d} x^{3}\right|_{\text {sub }}$ to the NFW profile of the host halo, $\mathrm{d} \tilde{N} /\left.\mathrm{d} x^{3}\right|_{\text {NFW }}$, which we also write as a function of $x$ and normalize to unity at $r_{\text {vir }}$. The 'bias function' is simply the ratio between $\mathrm{d} \tilde{N} /\left.\mathrm{d} x^{3}\right|_{\text {sub }}$ and $\mathrm{d} \tilde{N} /\left.\mathrm{d} x^{3}\right|_{\text {NFW }}$, which tends to unity when the subhalo distribution is unbiased with respect to the density profile of the host. We incorporate subhaloes

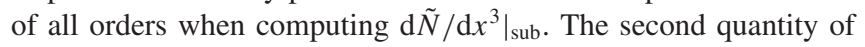
interest is the fraction of mass enclosed within a given projected host- 

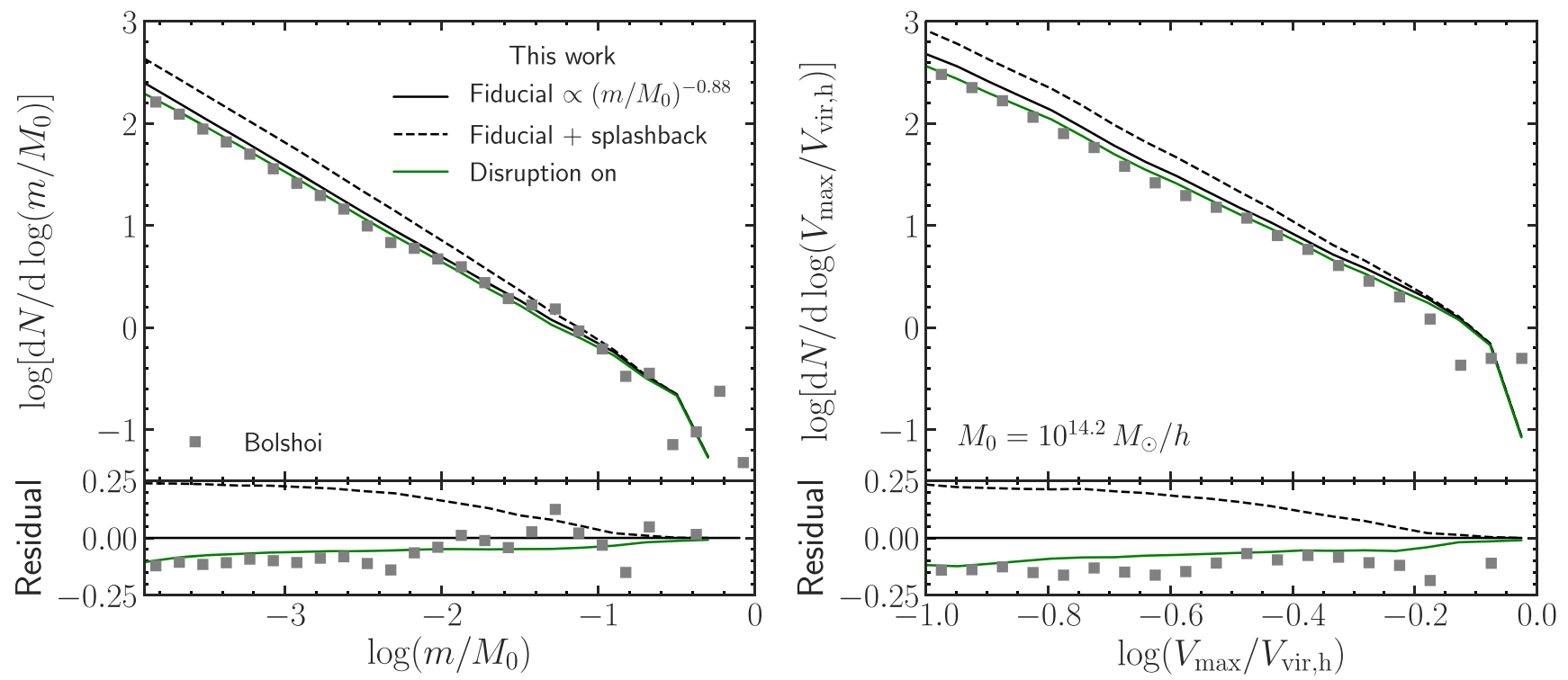

Figure 6. The SHMF (left-hand side) and subhalo maximum circular velocity function (SHVF; right-hand side) predictions for a host halo with $M_{0}=$ $10^{14.2} h^{-1} \mathrm{M}_{\odot}$ at $z=0$ (and virial velocity at $z=0$ denoted by $V_{\text {vir, h }}$ ). The SatGen results are averages taken over 10000 merger trees generated with $\psi_{\text {res }}=$ $10^{-4}$. These results are compared to the same quantities computed from 282 Bolshoi host haloes with $\log \left(M_{0} /\left[h^{-1} \mathrm{M}_{\odot}\right]\right) \in[14.0,14.5]$ (with a mean of 14.2), which are shown as grey squares. The fiducial SatGen predictions (black lines) are used as the baseline for comparison in the residual plots. In the 'fiducial + splashback' case (dashed black lines), we include subhaloes in the SHMF that are in the merger tree but instantaneously lie outside of the host $r_{\text {vir }}$ at $z=$ 0 . Lastly, the 'disruption on' case (green lines) demonstrates the impact of our artificial disruption mechanism (Section 2.4), which is calibrated to reproduce the statistical properties of Bolshoi subhalo disruption (D channel). At the low- $m / M_{0}$ end, artificial disruption suppresses the SatGen SHMF by $\sim 0.05-0.1$ dex, which brings our predictions into good agreement with Bolshoi. For $m / M_{0} \lesssim 10^{-2.5}$, nearly half of the subhaloes lie outside $r_{\text {vir }}$ (consistent with Bakels, Ludlow \& Power 2021).

centric radius that is bound in subhaloes. We define this quantity as

$F_{\text {sub }}(<X)=\frac{1}{M(<X)} \sum_{X_{i}<X} m_{i}$,

where $X=R / R_{\text {vir }}, R$ is the projected radius, the sum runs over all firstorder subhaloes (due to the inclusive mass definition) with projected radii within $R$, and $M(<R)$ is the projected mass profile of the NFW host halo (see Golse \& Kneib 2002). Finally, in Section 3.3, we focus on $f_{\text {sub }}\left(<r_{\text {vir }}\right)$, which is computed in the same way as $F_{\text {sub }}$ except that three-dimensional radii are used instead.

\subsubsection{Number density and radial bias profiles}

We begin by studying $\mathrm{d} \tilde{N} /\left.\mathrm{d} x^{3}\right|_{\text {sub }}$ and the corresponding bias function in Fig. 7. Since we aim to make direct comparisons to Bolshoi, we set $\psi_{\text {res }}=m_{\text {res, } \mathrm{B}} / M_{0}$, where $m_{\text {res, B }}=10^{9.83} h^{-1} \mathrm{M}_{\odot}$ corresponds to the 50-particle halo limit that we impose on the Bolshoi results. For $M_{0}=10^{14.2} h^{-1} \mathrm{M}_{\odot}$, this corresponds to $\log \left(\psi_{\text {res }}\right)=-4.37$. We compare the mean results obtained from 2000 SatGen trees with the mean of the 282 Bolshoi host haloes with $\log \left(M_{0} /\left[h^{-1} \mathrm{M}_{\odot}\right]\right) \in$ $[14.0,14.5]$. The shaded regions denote the $16-84$ percentiles of the halo-to-halo variance. The SatGen results are shown for three cases. The 'fiducial' result considers all subhaloes with $m / m_{\text {acc }} \geq \phi_{\text {res }}=$ $10^{-5}$, whereas the 'withering' result is limited to subhaloes with $m \geq \psi_{\text {res }} M_{0}$. Lastly, the 'withering + disruption' result includes the impact of the artificial disruption mechanism and thus can be compared directly to Bolshoi.

The radial profile of Bolshoi subhaloes becomes increasingly biased towards the central region of the host, something that has been pointed out in numerous previous studies (e.g. Diemand, Moore \& Stadel 2004; Springel et al. 2008; Han et al. 2016). The Bolshoi radial profile and bias function are reproduced exquisitely by SatGen, but only when the impact of both withering and artificial disruption are included. Modelling the simulation mass limit alone is sufficient to reproduce the Bolshoi mean curves within the halo-to-halo variance of SatGen; however, when artificial disruption is also taken into account, the mean curves are brought into near perfect agreement. Artificial disruption further suppresses the mean $\mathrm{d} \tilde{N} /\left.\mathrm{d} x^{3}\right|_{\text {sub }}$ by roughly a factor of 2 in the central region of the host. When all subhaloes can instead evolve down to $\mathrm{m} / \mathrm{m}_{\text {acc }}=10^{-5}$, regardless of $m_{\text {acc }}$, we find that the radial bias is completely eliminated. In fact, we obtain a slight overabundance of subhaloes towards the centre. This is due to DF, as we obtain a fully unbiased radial profile when we set $\beta_{\mathrm{DF}}=0$ (i.e. no DF). We note that Han et al. (2016) report a similar finding in the Aquarius simulations (Springel et al. 2008). By following the most-bound particle at accretion of all subhaloes (regardless of whether or not the subhalo survives to the present day), they find a DF-driven overabundance of subhalo remnants in the halo centre that decreases towards a fully unbiased profile as $m_{\mathrm{acc}}$ decreases. Taken together with SatGen, these results demonstrate that the chief cause of the dearth of subhaloes in the central regions of haloes is the limiting mass resolution of the simulation. It is neither physical nor primarily a manifestation of artificial disruption; the latter only makes a relatively modest impact.

\subsubsection{Projected enclosed substructure fraction}

Fig. 8 compares the $F_{\text {sub }}(<X)$ predictions of SatGen to the results of Bolshoi. ${ }^{11}$ We use the same SatGen data, simulation data, and

\footnotetext{
${ }^{11}$ Since the simulation halo catalogues are constructed such that subhaloes must be instantaneously located within the virial radius of their host, we also
} 

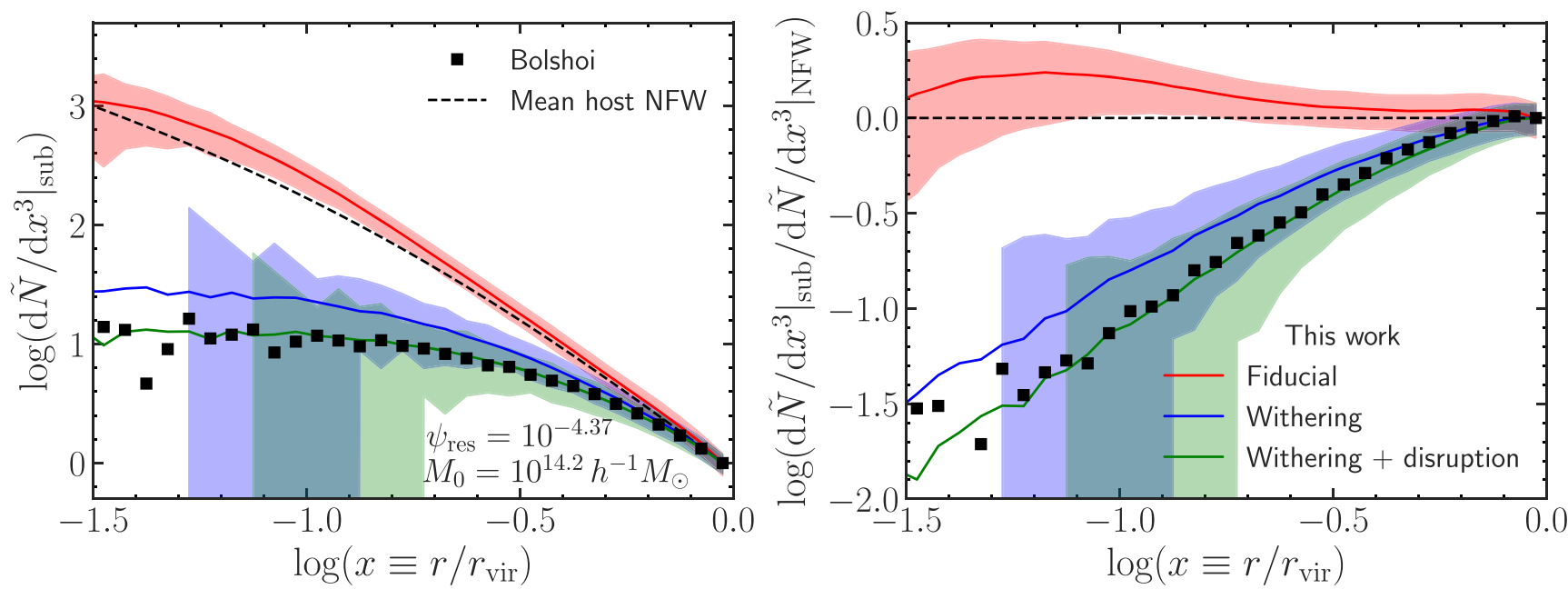

Figure 7. The subhalo radial profile (including subhaloes of all orders), $\mathrm{d} \tilde{N} /\left.\mathrm{d} x^{3}\right|_{\text {sub }}$ (left-hand side), normalized to unity at $r_{\text {vir }}$ and the bias function (right-hand side), which quantifies how the radial profile differs from the host density profile. The SatGen results are computed from 2000 merger trees of systems with $M_{0}=10^{14.2} h^{-1} \mathrm{M}_{\odot}$ at $z=0$ and a merger tree resolution limit of $\psi_{\text {res }}=10^{-4.37}$, consistent with the Bolshoi resolution limit for hosts of the same mass. The lines represent the sample means and the shaded regions denote the 16-84 percentiles taken over the sample, which quantify the halo-to-halo variance. The fiducial result (red) includes all subhaloes with $m / m_{\mathrm{acc}} \geq 10^{-5}$ (approximating the lack of a resolution limit), whereas the 'withering' result (blue) mimics the Bolshoi mass limit by only including subhaloes down to $m=\psi_{\text {res }} M_{0}$. Lastly, 'withering + disruption' (green) additionally includes the statistical treatment of artificial disruption (Section 2.4). The same quantities are computed from the 282 Bolshoi host haloes with $\log \left(M_{0} /\left[h^{-1} \mathrm{M}_{\odot}\right]\right) \in[14.0,14.5]$ (black squares). When artificial disruption and withering are taken into account, SatGen is able to exquisitely reproduce the Bolshoi bias function. In the absence of such numerical limitations, SatGen predicts a nearly unbiased radial profile (in agreement with Han et al. 2016).

plotting conventions as in Fig. 7, with the only difference being that the curves/points correspond to sample medians. Since the (projected) enclosed substructure mass fraction, $F_{\text {sub }}(<X)$, is simply a mass-weighted radial profile, and since SatGen reproduces both the SHMF and radial profile of Bolshoi subhaloes, it should come as little surprise that the model also succeeds at predicting $F_{\text {sub }}(<$ $X$ ). Once again, when we include the effects of both withering and artificial disruption, the model predictions are in nearly perfect agreement with Bolshoi. Without accounting for artificial disruption, the median Bolshoi $F_{\text {sub }}(<X)$ curve barely lies within the halo-tohalo variance of the withering-only prediction (for small $X$ ). Similar to $\mathrm{d} \tilde{N} /\left.\mathrm{d} x^{3}\right|_{\text {sub }}$, at $X \approx 0.1$, artificial disruption suppresses the median $F_{\text {sub }}$ by roughly a factor of 2 . The difference between the fiducial and withering-only model prediction is quite small, which lies in stark contrast to the number density profile. The reason for this is that the enhanced resolution of the fiducial model predominantly results in an increased abundance of highly stripped low- $m_{\text {acc }}$ subhaloes, which contribute little to the total substructure mass but make up a substantial portion of the number density. As we discuss in Section 3.3, the substructure mass fraction is primarily sensitive to the merger tree resolution $\left(\psi_{\text {res }}\right)$.

\subsubsection{Dependence of $\mathrm{d} \tilde{N} /\left.\mathrm{d} x^{3}\right|_{\text {sub }}$ on subhalo properties}

We have demonstrated that by properly modelling the effects of withering and artificial disruption on the subhalo population, SatGen can successfully reproduce the radial distribution of simulated subhaloes. We now take a closer look at the $\mathrm{d} \tilde{N} /\left.\mathrm{d} x^{3}\right|_{\text {sub }}$ predictions of our fiducial model in the absence of these numerical depletion channels. Here, we analyse the results of 2000 SatGen trees with

only consider SatGen subhaloes within the three-dimensional virial extent of the host halo when computing $F_{\text {sub }}(<X)$.

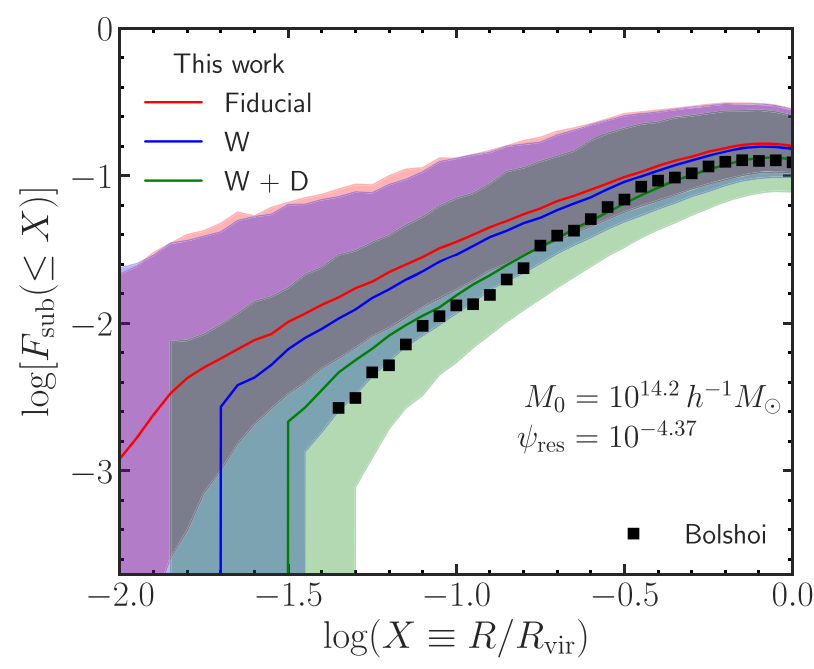

Figure 8. The fraction of mass enclosed within a given projected hostcentric radius that is bound in first-order subhaloes, $F_{\text {sub }}(<X)$, as defined in equation (15). The same SatGen predictions, Bolshoi results, and plotting conventions are used as in Fig. 7, with the exception being that the curves/points instead correspond to the sample medians. When both withering and artificial disruption are emulated, Sat Gen closely reproduces the Bolshoi $F_{\text {sub }}(<X)$ profile. The substructure mass fraction is only weakly enhanced by the additional resolution in $\mathrm{m} / \mathrm{m}_{\text {acc }}$-space afforded by the fiducial model, but it is reasonably sensitive to $\psi_{\text {res }}$ (see Section 3.3).

$M_{0}=10^{14.2} h^{-1} \mathrm{M}_{\odot}$ at $z=0$ and $\psi_{\text {res }}=\phi_{\text {res }}=10^{-5}$. Thus, the lowest $m_{\text {acc }}$ subhaloes are tracked all the way down to $10^{4.2} h^{-1} \mathrm{M}_{\odot}$. In Fig. 9, we plot the mean $\mathrm{d} \tilde{N} /\left.\mathrm{d} x^{3}\right|_{\text {sub }}$ computed using subhaloes from these trees binned by several properties: (i) $m_{\text {acc }} / M_{0}$, (ii) $m / m_{\text {acc }}$, (iii) $V_{\text {peak }} / V_{\text {vir, h }}$, where $V_{\text {peak }}$ is the peak $V_{\max }$ attained by the subhalo 

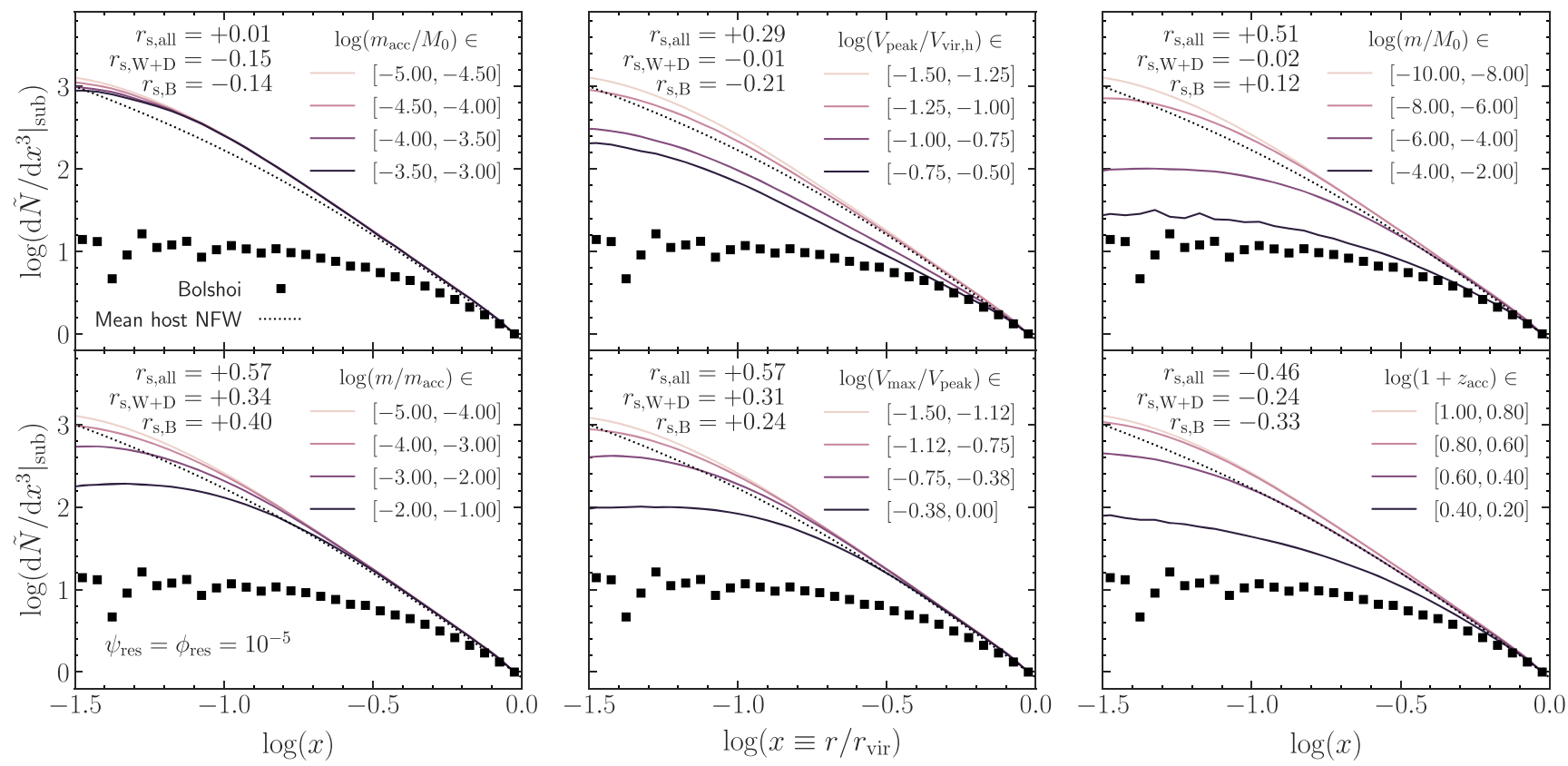

Figure 9. The subhalo radial profiles for 2000 SatGen trees with $M_{0}=10^{14.2} h^{-1} \mathrm{M}_{\odot}$ at $z=0$ and $\psi_{\text {res }}=\phi_{\text {res }}=10^{-5}$. In each panel, the subhaloes are binned by a different property and the mean $\mathrm{d} \tilde{N} /\left.\mathrm{d} x^{3}\right|_{\text {sub }}$ is computed for each bin. In order to assess the amount of bias, we plot the mean density profile of the host in each panel (dotted line). We overplot the mean Bolshoi radial profile of all subhaloes in each panel (black squares). We compute the Spearman coefficient between $r / r_{\mathrm{vir}}$ and each property for all subhaloes $\left(r_{\mathrm{s}, \text { all }}\right)$ and the subhaloes that would survive Bolshoi withering and artificial disruption $\left(r_{\mathrm{s}} \mathrm{W}+\mathrm{D}\right)$. There is little dependence on $m_{\text {acc }} / M_{0}$. As evidenced by the $m / m_{\text {acc }}$ and $V_{\max } / V_{\text {peak }}$ panels, highly stripped subhaloes follow the host density profile with little bias whereas minimally stripped systems are less commonly found in the halo centre. Similarly, massive, recently accreted subhaloes are biased towards the outer halo whereas older, less massive subhaloes are unbiased. Withering and artificial disruption tend to weaken (or reverse) the Spearman correlation between each property and $r / r_{\mathrm{vir}}$, bringing our $r_{\mathrm{s}, \mathrm{W}+\mathrm{D}}$ into good agreement with Bolshoi $\left(r_{\mathrm{s}, \mathrm{B}}\right.$, as computed in van den Bosch et al. 2016).

over its life (in SatGen, this is equivalent to the $V_{\max }$ at accretion, $V_{\text {max, acc }}$ ), (iv) $V_{\text {max }} / V_{\text {peak }}$, (v) $m / M_{0}$, and (vi) $\log \left(1+z_{\text {acc }}\right.$ ). For comparison, we also plot the mean Bolshoi $\mathrm{d} \tilde{N} /\left.\mathrm{d} x^{3}\right|_{\text {sub }}$ computed using all subhaloes (i.e. the same as in Fig. 7) in each panel. Lastly, in order to facilitate a comparison with the segregation study of van den Bosch et al. (2016), we also compute the Spearman rank correlation coefficient, $r_{\mathrm{s}}$, between $r / r_{\text {vir }}$ and each of the six properties computed with all subhaloes (denoted $r_{\mathrm{s} \text {, all }}$ ) and with subhaloes that would survive Bolshoi withering $\left(m>m_{\mathrm{res}, \mathrm{B}}\right)$ and artificial disruption (denoted $r_{\mathrm{s}, \mathrm{W}+\mathrm{D}}$, which can be directly compared to the Bolshoi results, $\left.r_{\mathrm{s}, \mathrm{B}}\right)$.

The normalized radial profile is nearly independent of $m_{\mathrm{acc}} / M_{0}$. On the other hand, systems with larger $V_{\text {peak }} / V_{\text {vir, h }}$ (i.e. systems with either larger $m_{\mathrm{acc}}$ or $c_{\mathrm{vir}, \mathrm{s}}$ ) tend to be biased towards the halo outskirts. van den Bosch et al. (2016) find the opposite, namely that Bolshoi subhaloes exhibit a weak, negative correlation between $r / r_{\text {vir }}$ and both $m_{\text {acc }} / M_{0}$ and $V_{\text {peak }} / V_{\text {vir, h. }}$. However, when both withering and artificial disruption are taken into account, we find that the sign of $r_{\mathrm{s}}$ flips from positive to negative for both properties, becoming consistent with Bolshoi. The $m / m_{\text {acc }}, m / M_{0}, V_{\text {max }} / V_{\text {peak }}$, and $\log (1$ $\left.+z_{\text {acc }}\right)$ panels all tell a similar story: older, less massive, and highly stripped subhaloes follow the host potential with minimal bias. However, recently accreted, massive, and minimally-stripped systems are biased towards the halo outskirts. These trends are weakened by withering and artificial disruption, bringing the $r_{\mathrm{s}, \mathrm{W}+\mathrm{D}}$ for each into good agreement with van den Bosch et al. (2016).

Taken together, SatGen predicts that the full subhalo population should exhibit little bias with respect to the host. The dearth of subhaloes in the halo centre, which is found universally in DM-only simulations (e.g. Ghigna et al. 1998; Springel et al. 2001; Diemand et al. 2004; Springel et al. 2008; Han et al. 2016), is a result of inadequate resolution that causes the non-physical elimination of old, highly stripped subhalo remnants that should be abundant in the host core.

\subsection{Substructure mass fractions}

We denote the fraction of matter bound into subhaloes within the virial radius of the host as $f_{\text {sub }}\left(<r_{\text {vir }}\right)$. In this section, we study how the SatGen predictions of $f_{\text {sub }}\left(<r_{\text {vir }}\right)$ vary with resolution limit, set by $\psi_{\text {res }}$, and how they are affected by artificial disruption. In what follows, we write $f_{\text {sub }}\left(\psi_{\text {res }}\right)$ to represent the value of $f_{\text {sub }}(<$ $r_{\text {vir }}$ ) computed from first-order subhaloes with $m>\psi_{\text {res }} M_{0}$. Written explicitly,

$f_{\text {sub }}\left(\psi_{\text {res }}\right)=\frac{1}{M_{0}} \sum_{\substack{r<r_{\mathrm{vir}} \\ m>\psi_{\mathrm{res}} M_{0}}} m_{i}$,

where the summation runs over first-order subhaloes only. We conclude the section by demonstrating that $f_{\text {sub }}$ is insensitive to small changes in the stripping efficiency parameter, $\alpha$, and the DF strength, $\beta_{\mathrm{DF}}$.

\subsubsection{Comparison of $\mathrm{f}_{\text {sub }}\left(\psi_{\text {res }}\right)$ to Bolshoi}

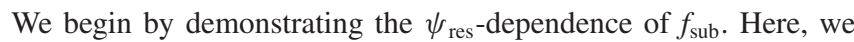
include artificial disruption in the SatGen predictions in order to facilitate comparisons with Bolshoi. In Fig. 10, we plot $f_{\text {sub }}\left(\psi_{\text {res }}\right)$ 


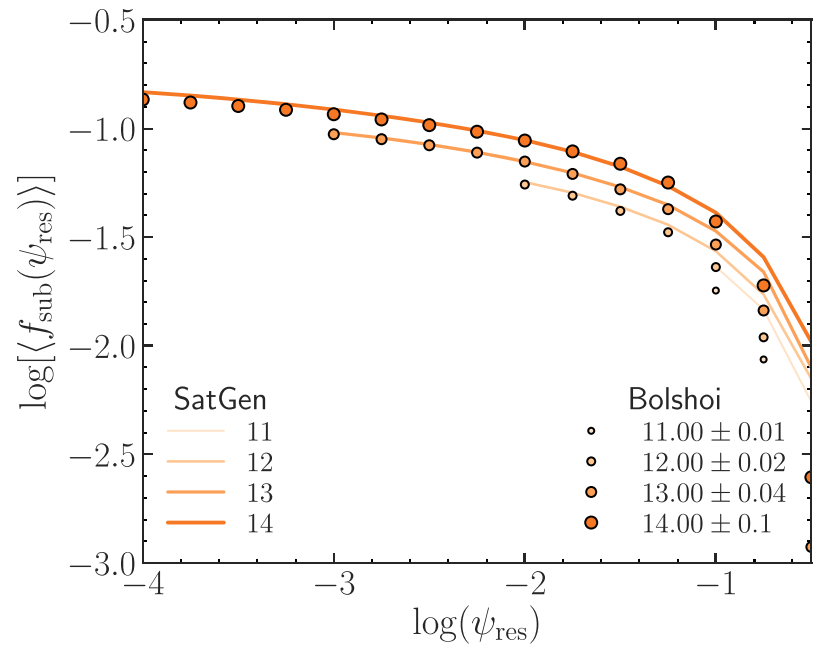

Figure 10. The average fraction of mass bound in subhaloes with $m>$ $\psi_{\text {res }} M_{0}$ within $r_{\text {vir }}$ of host haloes of a given $M_{0}$, as defined in equation (16). The SatGen predictions are generated using 10000 trees for each halo mass and are suppressed via the artificial disruption model (Section 2.4). The masses in the legend are reported in $\log \left(M_{0} /\left[h^{-1} \mathrm{M}_{\odot}\right]\right)$. We plot the curves down to the $\psi_{\text {res }}$ that corresponds to the Bolshoi 50-particle mass limit for each $M_{0}$. The model predictions agree well with the simulation results over a range of $M_{0}$.

for several different halo masses. The SatGen predictions are obtained using 10000 trees (with $\psi_{\text {res }}=10^{-4}$ ) of haloes with $M_{0}=$ $10^{11}-10^{14} h^{-1} \mathrm{M}_{\odot}$ at $z=0$. The Bolshoi results are computed from the $8815,4713,1138$, and 244 host haloes with $M_{0}=10^{11 \pm 0.01}$, $10^{12 \pm 0.02}, 10^{13 \pm 0.04}$, and $10^{14 \pm 0.1} h^{-1} \mathrm{M}_{\odot}$ at $z=0$. For each halo mass, we only show results down to the $\psi_{\text {res }}$ that corresponds to the 50-particle Bolshoi mass limit. As is evident, when combined with the Bolshoi-calibrated artificial disruption mechanism, SatGen is able to accurately reproduce the subhalo statistics (and their resolution dependence) of simulated haloes over several orders of magnitude in mass. There is some tension for $\psi_{\text {res }} \gtrsim 0.1$, indicating that the SHMFs of SatGen and Bolshoi disagree at the massive end. However, this likely reflects uncertainties with the (sub)halo finder used to analyse the simulation results rather than a shortcoming of SatGen (see van den Bosch \& Jiang 2016 for a detailed discussion).

\subsubsection{Mass-dependence and halo-to-halo variance of $\mathrm{f}_{\text {sub }}$}

Fig. 11 plots $f_{\text {sub }}\left(\psi_{\text {res }}=10^{-4}\right)$ as a function of host halo mass. These results have been obtained using 10000 trees each (with $\psi_{\text {res }}=10^{-4}$ ) for haloes with $\log \left(M_{0} /\left[h^{-1} \mathrm{M}_{\odot}\right]\right) \in[11,15]$ at $z=0$. Note that we have not included our treatment of artificial disruption here and the results are thus intended to reflect estimates of the true subhalo mass fractions in the absence of numerical artefacts. The left-hand panel shows the mean, median and 16-84percentiles for both first- and second-order subhaloes, as indicated, whereas the right-hand panel plots the corresponding cumulative distribution functions.

Overall, the trends shown are consistent with the orbit-averaged model used by Jiang \& van den Bosch (2017): $f_{\text {sub }}$ increases with $M_{0}$ and the halo-to-halo variance decreases slightly with $M_{0}$. As discussed in detail in Jiang \& van den Bosch (2017), this halo-tohalo variance is predominately driven by variance in the halo mass accretion histories (see also e.g. Giocoli et al. 2010; Green et al. 2020). The second-order $f_{\text {sub }}$ also increases with $M_{0}$, has much larger $\log$-scatter than the total $f_{\text {sub }}$, and its mean is smaller by a factor of $\approx 15-30$. This difference between first- and second-order $f_{\text {sub }}$ is considerably larger than predicted by Jiang \& van den Bosch (2017), which is primarily due to the fact that SatGen allows higher order subhaloes to be stripped from their parent subhalo (see Section 2.3.3).

For comparison, we also plot the result of Okabe et al. (2014), who used weak gravitational lensing to infer $f_{\text {sub }}=0.226_{-0.085}^{+0.111}$ for the Coma cluster, ${ }^{12}$ which is assumed to have a mass of $M_{0}=8.92_{-5.17}^{+20.05} \times 10^{14} h^{-1} \mathrm{M}_{\odot}$ (Okabe, Okura \& Futamase 2010). Our SatGen predictions are in excellent agreement with this measurement, demonstrating consistency between observations and the $\Lambda$ CDM paradigm.

\subsubsection{Impact of disruption on $\mathrm{f}_{\text {sub }}$}

Overall, the results of previous subsections illustrate that artificial disruption impacts subhalo statistics less significantly than the factor of 2 suggested by GB19. We now formalize this by comparing SatGen predictions of $f_{\text {sub }}\left(\psi_{\text {res }}=m_{\text {res, } \mathrm{B}} / M_{0}\right)$ with and without the impact of artificial disruption included (but with the same degree of withering in both cases since $\psi_{\text {res }}$ is fixed). For this test, we use 10000 trees with $\psi_{\text {res }}=10^{-4}$ and $M_{0}=10^{12}$ and $10^{13} h^{-1} \mathrm{M}_{\odot}$ as well as 2000 trees with $\psi_{\text {res }}=10^{-5}$ and $M_{0}=10^{14.2} h^{-1} \mathrm{M}_{\odot}$ in order to estimate $f_{\text {sub }}\left(\psi_{\text {res }}=m_{\text {res, }} / M_{0}\right)$ with and without disruption. ${ }^{13}$ We find that artificial disruption results in a relative suppression of $f_{\text {sub }}\left(\psi_{\text {res }}=\right.$ $\left.m_{\text {res, } \mathrm{B}} / M_{0}\right)$ by 8,10 , and 12 per cent for $M_{0}=10^{12}, 10^{13}$, and $10^{14.2} h^{-1} \mathrm{M}_{\odot}$, respectively. Indeed, this level of suppression is significantly less than a factor of 2 (i.e. 50 per cent). As already discussed in Section 3.1, the primary reason that the GB19 estimate of the artificial disruption impact is much larger is that the orbit-averaged model on which their estimate is based does not account for splashback haloes (i.e. the fact that at any moment in time about half of all haloes ever accreted by the host are located outside of the virial radius).

\subsubsection{Insensitivity of $\mathrm{f}_{\text {sub }}$ to the model parameter choices}

The substructure mass fraction is a useful summary statistic for illustrating how sensitive SatGen is to our model parameters (the stripping efficiency, $\alpha$, and the DF strength, $\left.\beta_{\mathrm{DF}}\right)$. For this test, we once again focus on $M_{0}=10^{14.2} h^{-1} \mathrm{M}_{\odot}$ haloes. We use 10000 trees with $\psi_{\text {res }}=10^{-4}$ and evolve the subhaloes using each of the following cases: (i) our fiducial parameters $\left(\beta_{\mathrm{DF}}=0.75\right.$ and $\alpha=\alpha\left(c_{\mathrm{vir}, \mathrm{s}} / c_{\mathrm{vir}, \mathrm{h}}\right)$ described by equation [11]), (ii) fiducial $\beta_{\mathrm{DF}}=0.75$ and fixed $\alpha=0.6$, and (iii) fiducial $\alpha=\alpha\left(c_{\mathrm{vir}, \mathrm{s}} / c_{\mathrm{vir}, \mathrm{h}}\right)$ and the 'natural' $\beta_{\mathrm{DF}}=1.0$ (i.e. Chandrasekhar DF without a correction factor). As our benchmark, we consider the fractional change in the mean $f_{\text {sub }}\left(\psi_{\text {res }}=10^{-4}\right)$ relative to the fiducial case. Setting $\alpha=0.6$ results in a 2 per cent relative increase in $f_{\text {sub }}$ relative to fiducial. Increasing $\beta_{\mathrm{DF}}$ from 0.75 to 1.0 results in an $\sim 4$ per cent relative decrease in $f_{\text {sub. }}$. The level of impact on other statistics (i.e. SHMF, radial profiles) is comparable. Hence, we conclude that our model predictions are reliable at the level of a few percent and that the uncertainties are small in comparison to the halo-to-halo variance. The sensitivity to these parameters is also significantly smaller than the impact of artificial disruption on

\footnotetext{
${ }^{12}$ This subhalo mass fraction is measured with $\psi_{\text {res }}=10^{-3}$, rather than $10^{-4}$. As shown in Fig. 10, the mean $f_{\text {sub }}\left(\psi_{\text {res }}=10^{-3}\right)$ is $\lesssim 0.1$ dex smaller than $f_{\text {sub }}\left(\psi_{\text {res }}=10^{-4}\right)$ for high-mass host haloes, which is negligible compared to both the halo-to-halo variance and the measurement error.

${ }^{13}$ Note that we need additional resolution for the high-mass case in order to resolve the merger trees down to $m_{\text {res, B }}$.
} 

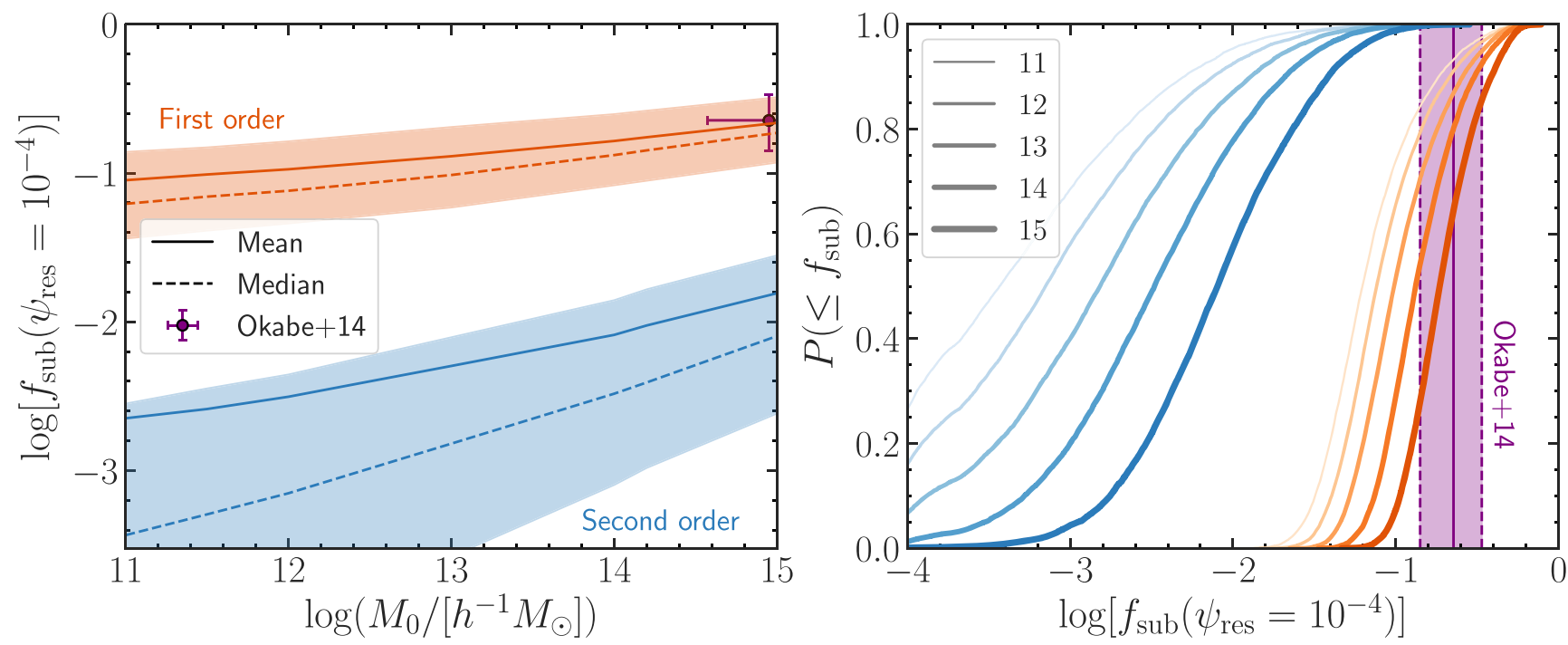

Figure 11. The total (first-order) and second-order $f_{\text {sub }}\left(\psi_{\text {res }}=10^{-4}\right)$ predictions of SatGen in the absence of artificial disruption. The mean, median, and 16-84percentile halo-to-halo variance of $f_{\text {sub }}$ (left-hand side) as well as the corresponding cumulative distribution function (right-hand side) are computed using 10000 trees of haloes with $M_{0}=10^{11}-10^{15} h^{-1} \mathrm{M}_{\odot}$ at $z=0$. The masses in the legend are reported in $\log \left(M_{0} /\left[h^{-1} \mathrm{M}_{\odot}\right]\right)$. Due to the inclusive mass definition, the first-order $f_{\text {sub }}$ includes the mass of subhaloes of all orders, whereas the second-order $f_{\text {sub }}$ includes the mass of subhaloes of order- 2 and higher. For comparison, we plot the gravitational lensing estimate of $f_{\text {sub }}\left(\psi_{\text {res }}=10^{-3}\right)$ for the Coma cluster measured by Okabe et al. (2014), finding excellent agreement with our model predictions.

the results of cosmological simulations, making SatGen a more reliable alternative for studying the substructure of DM haloes.

\subsection{Total $\mathrm{W}+\mathrm{D}$ disruption rate}

The artificial disruption mechanism of Section 2.4 is constructed such that the D channel population of SatGen subhaloes has an $f_{\text {dis }}$ distribution consistent with that of Bolshoi. However, this alone is not sufficient to guarantee that the $\mathrm{W}+\mathrm{D}$ numerical disruption rate of SatGen subhaloes is in agreement with the 13 per cent/Gyr that van den Bosch (2017) measured from the $\mathrm{W}+\mathrm{D}$ channel Bolshoi subhaloes. In order to make a fair comparison between the SatGen W + D disruption rate and Bolshoi, we run the following test. Starting with the same sample of Bolshoi host halo masses (at $z \sim 0$ ) as used in van den Bosch (2017), we randomly sub-sample 40000 masses from the total of $\sim 160000$. Rather than use a fixed $\psi_{\text {res }}$, we instead set $\psi_{\text {res }}=m_{\text {res, } \mathrm{B}} / M_{0}$, where $m_{\text {res, B }}=10^{9.83} h^{-1} \mathrm{M}_{\odot}$ is the 50-particle Bolshoi resolution mass. Following the procedure of van den Bosch (2017), we determine the $\mathrm{W}+\mathrm{D}$ disruption rate by measuring the fraction of the subhaloes present at $z=0.0174$ (i.e. $240 \mathrm{Myr}$ ago) that have been disrupted (either via the W or D channel) by $z=0$. In particular, our $z=0.0174$ sample consists of all subhaloes that have merged with the host, have a mass above both $m_{\text {res, B }}$ and the assigned $m_{\text {dis }}$ (i.e. it has neither disrupted nor withered by $z=0.0174$ ), and have an instantaneous orbital radius within $r_{\mathrm{vir}}$ of the host centre.

The subset of this sample with a $z=0$ mass below either $m_{\mathrm{res}, \mathrm{B}}$ or its assigned $m_{\text {dis }}$ are counted as having numerically disrupted between $z=0.0174$ and $z=0$. We convert this disruption fraction into a rate by dividing it by the $240 \mathrm{Myr}$ time interval considered. Using this approach, we determine that the combination of withering and our artificial disruption mechanism yields a $\mathrm{W}+\mathrm{D}$ numerical disruption rate of $\sim 16.7$ per cent/Gyr, which is only slightly larger than the 13 per cent/Gyr that van den Bosch (2017) measured in Bolshoi.
Hence, we conclude that our implementation of artificial disruption in SatGen accurately reproduces this numerical artefact in the Bolshoi simulation. However, we caution that it may not adequately describe artificial disruption in other simulations, each of which is likely to have subtly different disruption statistics. The real strength of SatGen is not its ability to reproduce the results of cosmological simulations but rather to make reliable predictions that are free from the numerical limitations that hamper such simulations.

\section{SUMMARY AND DISCUSSION}

This work represents the culmination of several previous studies aimed at quantifying the impact of artificial disruption on state-ofthe-art DM-only cosmological simulations. Studying the evolution of Bolshoi subhaloes, van den Bosch (2017) found that the combined effect of the finite mass resolution (i.e. withering) and artificial disruption results in rapid depletion of the subhalo population. In the follow-up studies of van den Bosch et al. (2018) and van den Bosch \& Ogiya (2018), the authors used a combination of analytical arguments and idealized numerical experiments to demonstrate that complete physical disruption of $\Lambda \mathrm{CDM}$ subhalo remnants is exceedingly rare, concluding that the majority of disruption seen in cosmological simulations must be numerical in nature. Following this, Ogiya et al. (2019) released the $D A S H$ library of high-resolution idealized simulations of halo mergers. This data release marked the beginning of a research program focused on developing a new semianalytical model of subhalo evolution that is calibrated independently of cosmological simulations, enabling its predictions to be free of the effects of artificial disruption. Thus, GB19 used DASH to construct an accurate model of the ESHDP, which is a simple function of the initial profile and the fraction of mass lost since infall (similar to the approaches of, e.g. Hayashi et al. 2003; Peñarrubia et al. 2010; Drakos et al. 2017; Errani \& Navarro 2020). Additionally, using the orbit-averaged subhalo evolution model and artificial 
disruption mechanism of Jiang \& van den Bosch (2016), GB19 inferred that artificial disruption could potentially be responsible for suppressing the SHMF normalization by as much as a factor of 2. Recently, Jiang et al. (2021) released the SatGen library, a new semi-analytical modelling framework for studying subhalo and satellite galaxy evolution in a full dynamical context (i.e. the orbits of individual subhaloes are integrated instead of using an orbit-averaged approach).

In the present paper, we used SatGen as a scaffolding to develop a comprehensive model of substructure evolution that is not adversely impacted by the limitations of artificial disruption and simulation resolution limits. To this end, we made several modifications and improvements to SatGen, which we summarize as follows:

(i) The initial orbits of infalling subhaloes are sampled using the state-of-the-art model of Li et al. (2020) (see Appendix A). This model marks an improvement over previous approaches (e.g. Zentner et al. 2005; Wetzel 2011; Jiang et al. 2015) because it is expressed as a general function of the host halo peak height and host-to-subhalo mass ratio. Furthermore, the free parameters of the model were fit using a large simulation suite.

(ii) The ESHDPs are characterized using the model of GB19 (Section 2.3.1). At infall, subhaloes are assumed to have NFW profiles. However, as mass is stripped and $\mathrm{m} / \mathrm{m}_{\text {acc }}$ decreases, the profile becomes tidally truncated in a manner consistent with the evolution of DASH subhaloes.

(iii) In line with the original SatGen implementation, the instantaneous subhalo mass-loss rate (Section 2.3.2) is written according to equation (7), which depends on the King (1962) tidal radius (computed using the ESHDPs), the local dynamical time, and the 'stripping efficiency', $\alpha$. We recalibrated $\alpha$ (equation [11]) so that the mass-loss model accurately reproduces the $m(t) / m_{\text {acc }}$ trajectories of DASH subhaloes.

(iv) The strength of the (Chandrasekhar) DF is controlled by a correction factor, $\beta_{\mathrm{DF}}$, which we calibrate such that SatGen reproduces the $m_{\text {acc }} / M_{0}$-dependence of the $\left\langle r / r_{\text {vir }}\right\rangle-z_{\text {acc }}$ relation of Bolshoi subhaloes (Section 2.5). We have demonstrated that the resulting best-fitting value $\left(\beta_{\mathrm{DF}}=0.75\right)$ is not adversely affected by artificial disruption in the Bolshoi simulation.

(v) In order to assess the impact of artificial disruption on simulations, we developed a model that reproduces the statistical properties of disruption in Bolshoi that can be optionally applied to SatGen results. We found that the $f_{\text {dis }}$ distribution of disrupted (D channel) Bolshoi subhaloes is well described by a family of lognormal distributions, the parameters of which are functions of $m_{\text {acc }}$ (Section 2.4).

(vi) SatGen is ideally suited to assess the impact of the resolution limit of numerical simulations by only including subhaloes with a final mass that lies above the merger tree resolution (i.e. $m>\psi_{\text {res }} M_{0}$ ). In addition, by instead allowing each subhalo to evolve down to arbitrary $\phi_{\text {res }}=m / m_{\text {acc }}$ (here, we have used values as low as $\phi_{\text {res }}=$ $10^{-5}$ ), SatGen can model the subhalo population with an effectively 'arbitrary resolution'.

We used this updated model to predict subhalo mass and maximum circular velocity functions, number density profiles, radial bias profiles, and substructure mass fractions. We considered the effect of both the simulation mass limit and artificial disruption on each quantity and studied the dependence of $f_{\text {sub }}$ on host halo mass. We summarize our most notable findings as follows: (i) When the effects of both withering and artificial disruption are included, SatGen yields subhalo demographics in excellent agreement with Bolshoi.

(ii) Artificial disruption only results in an $\sim 8-12$ per cent suppression of $f_{\text {sub }}\left(<r_{\text {vir }}\right)$ and an $\sim 20$ per cent suppression of the SHMF. While still significant, this greatly ameliorates previous concerns that the overall abundance of DM subhaloes could be artificially suppressed by a factor of 2 . However, the impact of artificial disruption is more pronounced at smaller host-centric radii, where it halves both $F_{\text {sub }}(<X)$ and $\mathrm{d} \tilde{N} /\left.\mathrm{d} x^{3}\right|_{\text {sub }}$ within $\sim 0.1 r_{\text {vir }}$.

(iii) By comparing the SHMF computed by including only subhaloes within $r_{\text {vir }}$ (i.e. consistent with simulation approaches) to the SHMF computed by including all surviving subhaloes ever accreted by the host, we infer that splashback haloes make up roughly half of the total subhalo population. This is in good agreement with results from several simulation studies (e.g. Gill et al. 2004; Ludlow et al. 2009; Bakels et al. 2021). Hence, it is essential that semianalytical models of subhalo and satellite galaxy evolution properly account for the splashback population. This is naturally achieved with full dynamical models, such as SatGen, which integrate the orbits of individual subhaloes. At the same time, it indicates a serious limitation of orbit-averaged approaches, such as those used in van den Bosch et al. (2005b) and Jiang \& van den Bosch (2016).

(iv) We have demonstrated that the radial bias in the subhalo number density (i.e. the dearth of subhaloes in the halo centre relative to the host density profile), a feature that is consistently present in DM-only simulations (e.g. Ghigna et al. 1998; Springel et al. 2001; Diemand et al. 2004; Springel et al. 2008; Han et al. 2016), is predominantly an artefact of the simulation mass resolution (at least in the absence of baryonic processes) and not of artificial disruption. The latter only slightly enhances the bias and is subdominant to the impact of the mass resolution. By allowing subhaloes to evolve down to arbitrarily low $\mathrm{m} / \mathrm{m}_{\text {acc }}$ (as opposed to having a fixed absolute mass limit), the radial bias is completely eliminated. In fact, DF causes a slight enhancement of the subhalo number density relative to the host profile near the halo centre, which marks a complete reversal of the trend seen in simulations.

Although the model presented here is able to accurately reproduce the subhalo statistics of den Bosch et al. (2005b) and Jiang \& van den Bosch cosmological simulation when its numerical limitations are properly taken into account, the true strength of the updated version of SatGen presented here lies in the fact that it can be used to predict subhalo demographics with an arbitrarily high resolution and in the absence of artificial disruption. We have therefore made the updated code publicly available in the hope that it will enable/accommodate a wide variety of future research programs. For example, SatGen could prove a powerful tool to investigate claimed discrepancies between simulations and observations regarding the abundance and central concentration of DM substructure (e.g. Carlsten et al. 2020; Meneghetti et al. 2020) and/or the DM deficiency of associated satellite galaxies (e.g. Ogiya 2018; Jackson et al. 2021).

\section{ACKNOWLEDGEMENTS}

The authors thank Uddipan Banik, Nicole Drakos, Dhruba Dutta Chowdhury, Zhaozhou Li, and Go Ogiya for helpful conversations throughout the development of this work. SBG is supported by the US National Science Foundation Graduate Research Fellowship under Grant No. DGE-1752134. FCvdB is supported by the National Aeronautics and Space Administration through Grant No. 17-ATP170028 issued as part of the Astrophysics Theory Program. FJ is 
supported by the Troesh Fellowship from the California Institute of Technology.

\section{DATA AVAILABILITY}

The DASH simulation data is available online. ${ }^{14}$ The updated SatGen library is available in the sheridan branch of the SatGen GitHub repository. ${ }^{15}$

\section{REFERENCES}

Aung H., Nagai D., Rozo E., García R., 2021, MNRAS, 502, 1041

Bakels L., Ludlow A. D., Power C., 2021, MNRAS, 501, 5948

Behroozi P. S., Wechsler R. H., Conroy C., 2013, ApJ, 770, 57

Benson A. J., 2017, MNRAS, 467, 3454

Benson A. J., 2020, MNRAS, 493, 1268

Benson A. J., Frenk C. S., Baugh C. M., Cole S., Lacey C. G., 2001, MNRAS, 327, 1041

Berlind A. A. et al., 2003, ApJ, 593, 1

Binney J., Tremaine S., 2008, Galactic Dynamics, 2nd edn. Princeton University Presss, Princeton

Bonaca A. et al., 2020, ApJ, 892, L37

Bose S. et al., 2017, MNRAS, 464, 4520

Boylan-Kolchin M., Springel V., White S. D. M., Jenkins A., 2010, MNRAS, 406, 896

Bryan G. L., Norman M. L., 1998, ApJ, 495, 80

Bullock J. S., Kolatt T. S., Sigad Y., Somerville R. S., Kravtsov A. V., Klypin A. A., Primack J. R., Dekel A., 2001, MNRAS, 321, 559

Burkert A., 2000, ApJ, 534, L143

Campbell D., van den Bosch F. C., Padmanabhan N., Mao Y.-Y., Zentner A. R., Lange J. U., Jiang F., Villarreal A., 2018, MNRAS, 477, 359

Carlberg R. G., 2012, ApJ, 748, 20

Carlsten S. G., Greene J. E., Peter A. H. G., Greco J. P., Beaton R. L., 2020, ApJ, 902, 124

Chandrasekhar S., 1943, ApJ, 97, 255

Chaves-Montero J., Angulo R. E., Schaye J., Schaller M., Crain R. A., Furlong M., Theuns T., 2016, MNRAS, 460, 3100

Cole S., Lacey C. G., Baugh C. M., Frenk C. S., 2000, MNRAS, 319, 168

Colín P., Avila-Reese V., González-Samaniego A., Velázquez H., 2015, ApJ, 803,28

Conroy C., Wechsler R. H., Kravtsov A. V., 2006, ApJ, 647, 201

Dalal N., Kochanek C. S., 2002, ApJ, 572, 25

Delos M. S., 2019, Phys. Rev. D, 100, 063505

Diemand J., Moore B., Stadel J., 2004, MNRAS, 352, 535

Diemand J., Kuhlen M., Madau P., 2007, ApJ, 667, 859

Diemer B., 2020, ApJS, 251, 17

Diemer B., 2021, ApJ, 909, 112

Drakos N. E., Taylor J. E., Benson A. J., 2017, MNRAS, 468, 2345

Drakos N. E., Taylor J. E., Benson A. J., 2020, MNRAS, 494, 378

Erkal D., Belokurov V., Bovy J., Sanders J. L., 2016, MNRAS, 463, 102

Errani R., Navarro J. F., 2020, preprint (arXiv:2011.07077)

Facchinetti G., Lavalle J., Stref M., 2020, preprint (arXiv:2007.10392)

Fong M., Han J., 2021, MNRAS, Available at: https://doi.org/10.1093/mnra s/stab259

Gan J., Kang X., van den Bosch F. C., Hou J., 2010, MNRAS, 408, 2201

Gao L., White S. D. M., Jenkins A., Stoehr F., Springel V., 2004, MNRAS, 355,819

Gao L., Navarro J. F., Frenk C. S., Jenkins A., Springel V., White S. D. M., 2012, MNRAS, 425, 2169

Garrison-Kimmel S. et al., 2017, MNRAS, 471, 1709

Ghigna S., Moore B., Governato F., Lake G., Quinn T., Stadel J., 1998, MNRAS, 300, 146

Gill S. P. D., Knebe A., Gibson B. K., 2004, MNRAS, 351, 399

\footnotetext{
${ }^{14}$ https://cosmo.oca.eu/dash/

${ }^{15} \mathrm{https}: / /$ github.com/shergreen/SatGen/tree/sheridan
}

Gilman D., Birrer S., Treu T., 2020, A\&A, 642, A194

Giocoli C., Tormen G., Sheth R. K., van den Bosch F. C., 2010, MNRAS, 404, 502

Golse G., Kneib J. P., 2002, A\&A, 390, 821

Green S. B., van den Bosch F. C., 2019, MNRAS, 490, 2091 (GB19)

Green S. B., Aung H., Nagai D., van den Bosch F. C., 2020, MNRAS, 496, 2743

Griffen B. F., Ji A. P., Dooley G. A., Gómez F. A., Vogelsberger M., O'Shea B. W., Frebel A., 2016, ApJ, 818, 10

Guo Q., White S., Li C., Boylan-Kolchin M., 2010, MNRAS, 404, 1111

Han J., Cole S., Frenk C. S., Jing Y., 2016, MNRAS, 457, 1208

Hayashi E., Navarro J. F., Taylor J. E., Stadel J., Quinn T., 2003, ApJ, 584, 541

Hayashi K., Ichikawa K., Matsumoto S., Ibe M., Ishigaki M. N., Sugai H., 2016, MNRAS, 461, 2914

Hearin A. P., Zentner A. R., Berlind A. A., Newman J. A., 2013, MNRAS, 433, 659

Hezaveh Y. D. et al., 2016, ApJ, 823, 37

Hiroshima N., Ando S., Ishiyama T., 2018, Phys. Rev. D, 97, 123002

Jackson R. A. et al., 2021, MNRAS, 502, 1785

Jiang F., van den Bosch F. C., 2014, MNRAS, 440, 193

Jiang F., van den Bosch F. C., 2016, MNRAS, 458, 2848

Jiang F., van den Bosch F. C., 2017, MNRAS, 472, 657

Jiang L., Cole S., Sawala T., Frenk C. S., 2015, MNRAS, 448, 1674

Jiang F., Dekel A., Freundlich J., van den Bosch F. C., Green S. B., Hopkins P. F., Benson A., Du X., 2021, MNRAS, 502, 621

Kampakoglou M., Benson A. J., 2007, MNRAS, 374, 775

Keeton C. R., Moustakas L. A., 2009, ApJ, 699, 1720

King I., 1962, AJ, 67, 471

Klypin A. A., Trujillo-Gomez S., Primack J., 2011, ApJ, 740, 102

Klypin A., Prada F., Yepes G., Heß S., Gottlöber S., 2015, MNRAS, 447, 3693

Knebe A. et al., 2013, MNRAS, 435, 1618

Knebe A., Arnold B., Power C., Gibson B. K., 2008, MNRAS, 386, 1029

Kravtsov A. V., Berlind A. A., Wechsler R. H., Klypin A. A., Gottlöber S., Allgood B., Primack J. R., 2004, ApJ, 609, 35

Lange J. U., van den Bosch F. C., Zentner A. R., Wang K., Villarreal A. S., 2019, MNRAS, 487, 3112

Li Z.-Z., Zhao D.-H., Jing Y. P., Han J., Dong F.-Y., 2020, ApJ, 905, 177

Lovell M. R., Frenk C. S., Eke V. R., Jenkins A., Gao L., Theuns T., 2014, MNRAS, 439, 300

Ludlow A. D., Navarro J. F., Springel V., Jenkins A., Frenk C. S., Helmi A., 2009, ApJ, 692, 931

Ludlow A. D., Schaye J., Bower R., 2019, MNRAS, 488, 3663

Mansfield P., Avestruz C., 2020, MNRAS, 500, 3309

Meneghetti M. et al., 2020, Science, 369, 1347

Mo H., van den Bosch F. C., White S., 2010, Galaxy Formation and Evolution. Cambridge Univ. Press, Cambridge

Nadler E. O. et al., 2020, ApJ, 893, 48

Nadler E. O. et al., 2021, Phys. Rev. Lett., 126, 091101

Nadler E. O., Mao Y.-Y., Green G. M., Wechsler R. H., 2019, ApJ, 873, 34

Navarro J. F., Frenk C. S., White S. D. M., 1997, ApJ, 490, 493

Necib L. et al., 2020, Nat. Astron., 4, 1078

Newton O., Cautun M., Jenkins A., Frenk C. S., Helly J. C., 2018, MNRAS, 479,2853

Ngan W. H. W., Carlberg R. G., 2014, ApJ, 788, 181

Ogiya G., 2018, MNRAS, 480, L106

Ogiya G., van den Bosch F. C., Hahn O., Green S. B., Miller T. B., Burkert A., 2019, MNRAS, 485, 189

Oguri M., Lee J., 2004, MNRAS, 355, 120

Okabe N., Okura Y., Futamase T., 2010, ApJ, 713, 291

Okabe N., Futamase T., Kajisawa M., Kuroshima R., 2014, ApJ, 784, 90

Onions J. et al., 2012, MNRAS, 423, 1200

Parkinson H., Cole S., Helly J., 2008, MNRAS, 383, 557

Peñarrubia J., Benson A. J., 2005, MNRAS, 364, 977

Peñarrubia J., Navarro J. F., McConnachie A. W., 2008, ApJ, 673, 226

Peñarrubia J., Benson A. J., Walker M. G., Gilmore G., McConnachie A. W., Mayer L., 2010, MNRAS, 406, 1290 
Pieri L., Bertone G., Branchini E., 2008, MNRAS, 384, 1627

Pullen A. R., Benson A. J., Moustakas L. A., 2014, ApJ, 792, 24

Read J. I., Wilkinson M. I., Evans N. W., Gilmore G., Kleyna J. T., 2006, MNRAS, 367, 387

Reddick R. M., Wechsler R. H., Tinker J. L., Behroozi P. S., 2013, ApJ, 771, 30

Rico J., 2020, Galaxies, 8, 25

Rocha M., Peter A. H. G., Bullock J. S., Kaplinghat M., Garrison-Kimmel S., Oñorbe J., Moustakas L. A., 2013, MNRAS, 430, 81

Shu Y. et al., 2015, ApJ, 803, 71

Somalwar J. J., Chang L. J., Mishra-Sharma S., Lisanti M., 2021, ApJ, 906, 57

Springel V. et al., 2008, MNRAS, 391, 1685

Springel V., White S. D. M., Tormen G., Kauffmann G., 2001, MNRAS, 328, 726

Strigari L. E., Koushiappas S. M., Bullock J. S., Kaplinghat M., 2007, Phys. Rev. D, 75, 083526

Taffoni G., Mayer L., Colpi M., Governato F., 2003, MNRAS, 341, 434

Taylor J. E., Babul A., 2001, ApJ, 559, 716

Taylor J. E., Babul A., 2004, MNRAS, 348, 811

Tollet É., Cattaneo A., Mamon G. A., Moutard T., van den Bosch F. C., 2017, MNRAS, 471, 4170

Tormen G., Bouchet F. R., White S. D. M., 1997, MNRAS, 286, 865

Trujillo-Gomez S., Klypin A., Primack J., Romanowsky A. J., 2011, ApJ, 742,16

Vale A., Ostriker J. P., 2006, MNRAS, 371, 1173

van den Bosch F. C., Jiang F., 2016, MNRAS, 458, 2870

van den Bosch F. C., Yang X., Mo H. J., Norberg P., 2005a, MNRAS, 356, 1233

van den Bosch F. C., Tormen G., Giocoli C., 2005b, MNRAS, 359, 1029

van den Bosch F. C., Jiang F., Hearin A., Campbell D., Watson D., Padmanabhan N., 2014, MNRAS, 445, 1713

van den Bosch F. C., Ogiya G., Hahn O., Burkert A., 2018, MNRAS, 474, 3043

van den Bosch F. C., Lange J. U., Zentner A. R., 2019, MNRAS, 488, 4984

van den Bosch F. C., 2017, MNRAS, 468, 885

van den Bosch F. C., Ogiya G., 2018, MNRAS, 475, 4066

van den Bosch F. C., Jiang F., Campbell D., Behroozi P., 2016, MNRAS, 455, 158

Vattis K., Toomey M. W., Koushiappas S. M., 2020, preprint (arXiv:2008.1 1577)

Vegetti S., Koopmans L. V. E., Auger M. W., Treu T., Bolton A. S., 2014, MNRAS, 442, 2017

Vogelsberger M., Zavala J., Loeb A., 2012, MNRAS, 423, 3740

Wetzel A. R., 2011, MNRAS, 412, 49

Yang S., Du X., Benson A. J., Pullen A. R., Peter A. H. G., 2020, MNRAS, 498, 3902

Zentner A. R., Bullock J. S., 2003, ApJ, 598, 49

Zentner A. R., Berlind A. A., Bullock J. S., Kravtsov A. V., Wechsler R. H., 2005, ApJ, 624, 505

Zentner A. R., Hearin A. P., van den Bosch F. C., 2014, MNRAS, 443, 3044

Zhao D. H., Jing Y. P., Mo H. J., Börner G., 2009, ApJ, 707, 354

\section{APPENDIX: INITIAL ORBITS}

Here, we describe our approach for initializing subhalo orbits. We specify the initial phase-space coordinates of the infalling subhalo as

$\left\{r, \theta, \phi, v_{r}, v_{\theta}, v_{\phi}\right\}=\left\{r_{\mathrm{vir}}, \theta, \phi,-v \cos \gamma, v \sin \gamma \cos \delta, v \sin \gamma \sin \delta\right\}$. (A1)

We assume that subhalo infall occurs isotropically, and therefore select an initial azimuthal angle, $\phi$, uniformly from $[0,2 \pi)$ and an initial polar angle, $\theta$, by sampling $\cos \theta$ uniformly from $[0,1)$. In order to determine the initial velocity vector, the degrees of freedom of which are the speed, $v$, the angle between the velocity vector and the (negative of the) position vector, $\gamma$, and an additional angle that sets the orientation of the orbital plane, $\delta$, we use the universal model of Li et al. (2020), which has been calibrated using a large suite of cosmological simulations.

For all first infall events (i.e. for a given subhalo, only considering the first time a subhalo enters into the host virial radius) aggregated across all of the simulations and over a wide range of redshift snapshots, Li et al. (2020) find that $u \equiv v / V_{\text {vir, h }}$ (here, $V_{\text {vir, h }}$ denotes the instantaneous virial velocity of the host) is well described by a universal lognormal distribution that is peaked near unity and is independent of subhalo mass and redshift, $z$ :

$p(u) \mathrm{d} u=\frac{1}{\sqrt{2 \pi} \sigma_{1}} \exp \left[-\frac{\ln ^{2}\left(u / \mu_{1}\right)}{2 \sigma_{1}^{2}}\right] \frac{\mathrm{d} u}{u}$.

Here, $\mu_{1}=1.2$ and $\sigma_{1}=0.2$. They also find that mergers with larger $M_{\mathrm{vir}}$ (instantaneous virial mass of the host) and/or $\xi \equiv m_{\mathrm{acc}} / M_{\mathrm{vir}}$ result in more radial subhalo orbits, which is mainly attributed to gravitational focusing. By rewriting the host mass, $M_{\text {vir }}$, in terms of its corresponding density peak height, $v \equiv \delta_{\mathrm{c}}(z) / \sigma\left(M_{\mathrm{vir}}\right)$, where $\delta_{\mathrm{c}}(z)$ is the critical overdensity of collapse and $\sigma^{2}(M)$ is the mass variance, the authors find that the distribution of infall angles is redshift-independent and only depends on $u, v$, and $\xi$. Specifically, $\cos ^{2} \gamma$ follows an exponential distribution,

$p\left(\cos ^{2} \gamma \mid u, \nu, \xi\right) \mathrm{d} \cos ^{2} \gamma=\frac{\zeta}{\mathrm{e}^{\zeta}-1} \exp \left(\zeta \cos ^{2} \gamma\right) \mathrm{d} \cos ^{2} \gamma$,

where

$$
\begin{aligned}
& \zeta=a_{0} \exp \left[-\frac{\ln ^{2}\left(u / \mu_{2}\right)}{2 \sigma_{1}^{2}}\right]+A(u+1)+B, \\
& A=a_{1} v+a_{2} \zeta^{c}+a_{3} v \zeta^{c}, \text { and } \\
& B=b_{0}+b_{1} \zeta^{c}
\end{aligned}
$$

and the best-fitting parameters are $\left(a_{0}, a_{1}, a_{2}, a_{3}, b_{0}, b_{1}, \mu_{2}, c\right)=$ $(0.89,0.3,-3.33,0.56,-1.44,9.60,1.04,0.43)$.

We use equations (A2)-(A4) to sample the initial $v$ and $\gamma$ for each subhalo at infall. In order to set the orientation of the orbital plane, we assume isotropy and therefore draw $\delta$ uniformly from $[0,2 \pi)$.

Using the $u$ distribution of $\mathrm{Li}$ et al. (2020) results in a substantial fraction of sampled orbits with initial orbital energies that lie above the maximum value sampled in the DASH simulations (corresponding to $x_{\mathrm{c}}=2$ ). This fraction has a slight dependence on the host concentration. For example, for $c_{\mathrm{vir}, \mathrm{h}}=10$, a total of 25 per cent of subhaloes have $x_{\mathrm{c}}>2$ at infall and 2 per cent are initially unbound (i.e. $v$ at infall is larger than the escape velocity). Fortunately, the performance of our DASH-calibrated ESHDP model and mass-loss prescription both exhibit minimal dependence on the orbital parameters. We emphasize that the combined impact of DF and the growth of the host potential results in continuous reduction of the subhalo orbital energy, lowering $x_{\mathrm{c}}$ over time. These effects also drive subhaloes that are initially unbound to eventually become bound after infall; thus, we include these initially unbound orbits in the SatGen subhalo population.

This paper has been typeset from a $\mathrm{T}_{\mathrm{E}} \mathrm{X} / \mathrm{L} \mathrm{T} \mathrm{T}_{\mathrm{E}} \mathrm{X}$ file prepared by the author. 